\title{
A Regioselective Synthesis of Novel Functionalized Organochalcogen Compounds by Chalcogenocyclofunctionalization Reactions Based on Chalcogen Halides and Natural Products
}

\author{
Maxim V. Musalov (D), Vladimir A. Potapov*(D), Vladimir A. Yakimov, Maria V. Musalova, Arkady A. Maylyan, \\ Sergey V. Zinchenko and Svetlana V. Amosova (D)
}

Citation: Musalov, M.V.; Potapov, V.A.; Yakimov, V.A.; Musalova, M.V.; Maylyan, A.A.; Zinchenko, S.V.; Amosova, S.V. A Regioselective Synthesis of Novel Functionalized Organochalcogen Compounds by Chalcogenocyclofunctionalization Reactions Based on Chalcogen Halides and Natural Products. Molecules 2021, 26, 3729. https:// doi.org/10.3390/molecules26123729

Academic Editor: Luana Bagnoli

Received: 1 June 2021

Accepted: 17 June 2021

Published: 18 June 2021

Publisher's Note: MDPI stays neutral with regard to jurisdictional claims in published maps and institutional affiliations.

Copyright: (c) 2021 by the authors. Licensee MDPI, Basel, Switzerland. This article is an open access article distributed under the terms and conditions of the Creative Commons Attribution (CC BY) license (https:/ / creativecommons.org/licenses/by/ $4.0 /)$.
A. E. Favorsky Irkutsk Institute of Chemistry, Siberian Division of The Russian Academy of Sciences, 1 Favorsky Str., 664033 Irkutsk, Russia; musalov_maxim@irioch.irk.ru (M.V.M.); yakimov@irioch.irk.ru (V.A.Y.); musalova@irioch.irk.ru (M.V.M.); maylyan@irioch.irk.ru (A.A.M.); svz@irioch.irk.ru (S.V.Z.); amosova@irioch.irk.ru (S.V.A.)

* Correspondence: v.a.potapov@mail.ru

\begin{abstract}
The regioselective synthesis of novel functionalized condensed organochalcogen compounds by chalcogenocyclofunctionalization reactions based on chalcogen halides and the natural products thymol and carvacrol has been developed. The reactions of selenium dibromide with allyl thymol and allyl carvacrol proceeded in methylene chloride at room temperature in the presence of $\mathrm{NaHCO}_{3}$ affording bis[(7-isopropyl-4-methyl-2,3-dihydro-1-benzofuran-2-yl)methyl] and bis[(4-isopropyl-7-methyl-2,3-dihydro-1-benzofuran-2-yl)methyl] selenides in 90-92\% yield. Similar sulfides were obtained in $70-72 \%$ yields by the reaction of sulfur dichloride in chloroform under reflux. Trihalotellanes containing the same organic moieties were synthesized from allyl thymol, allyl carvacrol and tellurium tetrachloride or tetrabromide in quantitative yields. Corresponding functionalized ditellurides were prepared in 91-92\% yields by the reduction of the trichlorotellanes with sodium metabisulfite in two-phase solvent system. The comparison of reactivity of sulfur, selenium and tellurium halides in chalcogenocyclofunctionalization and distinguishing features of each reaction were discussed.
\end{abstract}

Keywords: thymol; carvacrol; selenium dibromide; tellurium tetrachloride; tellurium tetrabromide; sulfur dichloride

\section{Introduction}

Natural products and their derivatives play an important role in the discovery of new drugs [1-5]. Many modern drugs have been developed from natural products, and synthesis of novel functionalized compounds based on natural products is promising in terms of the possible manifestation of biological activity.

The present work is devoted to regioselective synthesis of novel condensed functionalized organochalcogen compounds by chalcogenocyclofunctionalization reactions based on selenium dihalides, tellurium tetrahalides and sulfur dichloride and natural products, thymol (2-isopropyl-5-methylphenol) and carvacrol (5-isopropyl-2-methylphenol). Thymol and carvacrol are isomeriC-Natural monoterpenoid phenols, found in essential oils of Thymus vulgaris (thyme) and Origanum vulgare (oregano) and extracted from various other kinds of plants.

The thyme herb has been used in folk traditional medicine as a sedative and antiseptic since ancient times [6]. Ancient Egyptians used thyme for embalming. The ancient Greeks used it in their baths and burned it as incense in their temples, believing it was a source of courage. The spread of thyme throughout Europe was thought to be due to the Romans, as they used it to purify their rooms and to give an aromatic flavor to some food and liqueurs [7]. Its extract was used as a natural antibacterial gargle for sore throat and colds. 
Not only thymol and carvacrol derivatives, but these natural products themselves show various types of biological activity [8-18]. Thymol is widely used in the chemical industry and pharmacotherapy due to its antibiotic $[9,10]$, anticancer [11], insecticidal $[12,13]$ and antileishmanial [14] properties and general non-genotoxicity or cytotoxicity on human cells [15-17]. Besides, thymol is applied as an active antiseptic ingredient in some toothpastes, medicinal ointments and drugs for inhalation. This natural product is also proposed as an environmental-friendly, rapidly degrading and non-persisting pesticide [8].

Derivatives of thymol and carvacrol exhibit a variety of biological activities [18-37] including antibacterial [18,22-24], antifungal [27-29], antitubercular [32] and anticancer [35-37] properties (some examples of biologically active thymol and carvacrol derivatives are presented in Figure 1). Simplest derivatives of these natural products such as allyl ether of thymol and allyl-substituted thymol (4-allyl-2-isopropyl-5-methylphenol) display antibacterial activity [18].<smiles>C=CCOc1cc(C)ccc1C(C)C</smiles><smiles>C=CCc1cc(C(C)C)c(O)cc1C</smiles>

Antibacterial activity<smiles>C/C=C(/C)C(=O)OCC(O)(COC(C)=O)c1ccc(C)cc1O</smiles>

Anticancer activity<smiles>Cc1cc(C)c(S(=O)(=O)N/N=C/c2c(C)ccc(C)c2O)c(C)c1</smiles>

Anticancer activity<smiles>Cc1ccc(C(O)(COC(=O)C(C)C)COC(=O)C(C)C)c(O)c1</smiles>

Antibacterial activity<smiles>Cc1ccc(C(C)C)c(OCc2nnc(SCC(=O)Nc3ccc(S(N)(=O)=O)cc3)n2Cc2ccccc2)c1</smiles>

Anticancer activity<smiles>Cc1ccc(C(C)C)cc1OCC(=O)NNS(=O)(=O)c1ccc(Cl)cc1</smiles>

Antifungal activity

Figure 1. Known derivatives of thymol and carvacrol exhibiting antibacterial [18,22,23], anticancer [18,35-37] and antifungal [27] activities.

The discovery of the biological role of selenium gave a powerful impetus to the rapid development organoselenium chemistry, which is currently occupied an important place in chemical research. It is worth noting that organoselenium compounds and especially selenium-containing heterocycles show a variety of biological activities including antibacterial, antitumor, anti-inflammatory, neuroprotective and glutathione peroxidase-like actions [38-47].

In recent years, chalcogen-induced cyclization and especially selenocyclofunctionalization reactions have acquired particular importance as valuable tools for modern organic synthesis. The growing interest in selenocyclofunctionalization was demonstrated in excellent books and reviews [45-54].

Similar methodology of intramolecular halocyclization of alkenes containing nucleophilic functions which afford useful heterocyclic compounds including nitrogen heterocycles are well documented in the literature [55-57]. However, opportunities of selenocyclofunctionalization reactions have not yet been fully realized.

Addition products of selenenyl halides to alkenes are very reactive in nucleophilic substitution reactions since halogen atoms in $\beta$-haloorganyl selenides are activated by 
anchimeric assistance of the selenium atom [58]. The high anchimeric assistance effect of the selenium atom plays an important role in selenocyclofunctionalization reactions which include intramolecular nucleophilic substitution. This effect leads to considerable activation of halogen atoms to nucleophilic substitution and intramolecular cyclization reactions proceed very smoothly. It has been found that the anchimeric assistance effect of the selenium atom is approximately two orders of magnitude greater than the analogous effect of the sulfur and nitrogen atoms [58].

Previously, we first introduced selenium dihalides in organic synthesis for preparation of organoselenium compounds [59-61] and used these reagents for selenocyclofunctionalization reactions [62-69]. The application of selenium dihalides in selenocyclofunctionalization allows to carry out two heterocyclization processes in one molecule and to obtain selenides containing two heterocyclic moieties [62-69].

Previously we studied annulation reactions of selenium dihalides with allyl thymyl and allyl carvacryl ethers (Scheme 1) [70,71]. A novel methodology to accelerate annulation reactions leading to condensed selenium heterocycles was developed. The reactions of selenium dihalides with allyl thymyl and allyl carvacryl ethers (as well as with methyleugenol, allyl 1-naphthyl and 1-naphthyl propargyl ethers) were carried out in solvent systems $\mathrm{CH}_{2} \mathrm{Cl}_{2} / \mathrm{ROH}$ or $\mathrm{CHCl}_{3} / \mathrm{ROH}$. It was found that addition of alcohol to methylene chloride or chloroform considerably accelerated annulation reactions [70,71]. In solvent systems $\mathrm{CH}_{2} \mathrm{Cl}_{2} / \mathrm{MeOH}$ or $\mathrm{CHCl}_{3} / \mathrm{MeOH}$, the reactions proceeded as annulation-methoxylation affording condensed methoxylated heterocycles. In the presence of isopropanol, the reactions were not accompanied by alkoxylation giving condensed halogen-containing products (Scheme 1). The annulation-acetoxylation reaction of selenium dibromide with allyl carvacryl ether in the solvent system $\mathrm{CH}_{2} \mathrm{Cl}_{2}$ / acetic acid affording bicylic acetoxy derivative in $98 \%$ yield was also developed [71]. Novel condensed functionalized products, 2,3-dihydro1,4-benzoxaselenine derivatives, bearing methoxy, acetoxy and halogen-containing groups were synthesized in high yields from selenium dihalides and allyl thymyl and allyl carvacryl ethers using this novel methodology (Scheme 1) [70,71].<smiles>COCC1COc2c(C(C)C)ccc(C)c2[Se]1</smiles>

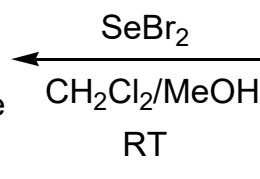

$90 \%$<smiles>COCC1COc2c(C)ccc(C(C)C)c2[Se]1</smiles><smiles>CC(O)(Br)CCCCC(O)(O)Br</smiles><smiles>C=CCOc1cc(C(C)C)ccc1C</smiles><smiles>[X]CC1COc2c(C(C)C)ccc(C)c2[Se]1</smiles>

$86-90 \%$

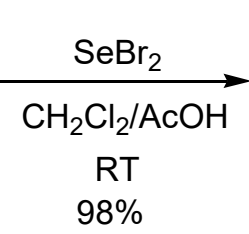<smiles>CC(=O)OCC1COc2c(C)ccc(C(C)C)c2[Se]1</smiles>

$\mathrm{X}=\mathrm{Cl}, \mathrm{Br}$

Scheme 1. Synthesis of novel condensed products by the annulation reactions of selenium dibromide with allyl carvacryl and allyl thymyl ethers.

\section{Results and Discussion}

The aim of this research is to develop an efficient regioselective synthesis of novel condensed functionalized organochalcogen compounds by chalcogenocyclofunctionalization reactions based on chalcogen halides and natural products thymol (1) and carvacrol (2). Starting compounds allyl ethers of thymol and carvacrol were prepared by the reaction of thymol and carvacrol with allylbromide in the presence of a base. Usually strong bases 
as sodium hydroxide or potassium carbonate are often used in alkylation reactions of phenols [72]. We developed efficient green chemistry synthesis of allyl thymyl ether 3 and allyl carvacryl ether 4 in near quantitative yields (97-98\%) at room temperature using environmentally tolerant sodium hydrocarbonate $\left(\mathrm{NaHCO}_{3}\right.$, baking soda, $\mathrm{E}$ number food additive code is E500) instead of strong bases like alkalis or carbonates (Scheme 2).<smiles>Cc1ccc(C(C)C)c(O)c1</smiles>

1<smiles>Cc1ccc(C(C)C)cc1O</smiles>

2
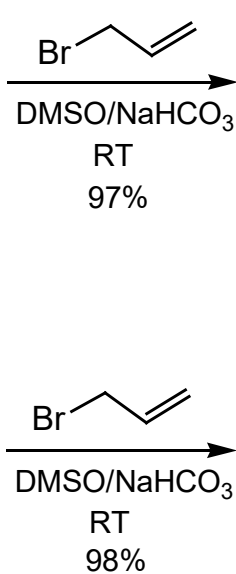

$98 \%$<smiles>C=CCOc1cc(C)ccc1C(C)C</smiles>

3<smiles>C=CCOc1cc(C(C)C)ccc1C</smiles>

4<smiles>C=CCc1c(C)ccc(C(C)C)c1O</smiles>

5

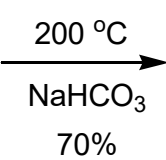<smiles>C=CCc1c(C(C)C)ccc(C)c1O</smiles>

6

Scheme 2. Synthesis of allyl thymol $\mathbf{5}$ and allyl carvacrol $\mathbf{6}$ by the reaction of thymol $\mathbf{1}$ and carvacrol 2 with allyl bromide followed by Claisen rearrangement of compounds 3 and 4 .

The conversion of allyl ethers $\mathbf{3}$ and $\mathbf{4}$ to corresponding allyl phenols $\mathbf{5}$ and $\mathbf{6}$ was developed using Claisen rearrangement (Scheme 2). It was established that the products 5 and 6 were formed in $75-80 \%$ yields when the reaction was carried out by heating allyl ethers 3 and 4 at $200{ }^{\circ} \mathrm{C}$ for $2 \mathrm{~h}$ in sealed tubes. The reaction is a [3,3]-sigmatropic rearrangement which leads to the formation of new carbon-carbon bond. It was found that an additive of sodium hydrocarbonate increases selectivity of the rearrangement. Pure products 5 and 6 were isolated by column chromatography on silica gel in 70-71\% yields (Scheme 2). Minor amounts of allyl ethers 3 and 4 remained unconverted in Claisen rearrangement, however, products $\mathbf{5}$ and $\mathbf{6}$ containing a polar hydroxyl group can be easily separated from them.

We studied the reactions of selenium dihalides with allyl thymol 5 and allyl carvacrol 6 and found that selenium dibromide is more efficient than selenium dichloride in selenocyclofunctionalization. The reactions smoothly proceeded in methylene chloride at room temperature. After completing the first stage (electrophilic addition of selenium dibromide to the double bond of allyl thymol $\mathbf{5}$ and allyl carvacrol $\mathbf{6}$ ), sodium hydrocarbonate as a mild base was added to the reaction mixture in order to catalyze intramolecular nucleophilic substitution of the bromine atom by the hydroxyl group with the formation of bis[(7isopropyl-4-methyl-2,3-dihydro-1-benzofuran-2-yl)methyl] and bis[(4-isopropyl-7-methyl2,3-dihydro-1-benzofuran-2-yl)methyl] selenides 7 and 8 in 90-92\% yield (Scheme 3). In the case of using selenium dichloride, the yields of products 7 and 8 were $67-70 \%$. 


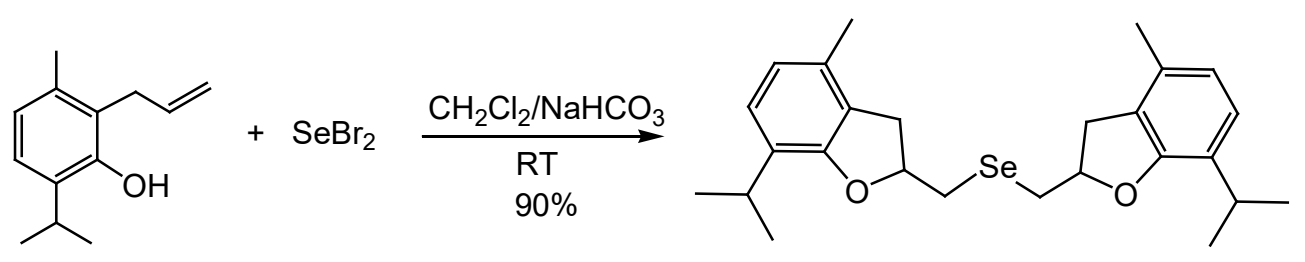

5<smiles>C=CCc1c(C(C)C)ccc(C)c1O</smiles><smiles>CC(CC=O)C(=O)O</smiles>

6<smiles>Cc1ccc(C(C)C)c2c1CC(C[Se]CC1Cc3c(C(C)C)ccc(C)c3O1)O2</smiles>

8

Scheme 3. Synthesis of selenides 7 and 8 by the reaction of selenium dibromide with allyl thymol 5 and allyl carvacrol 6.

The reaction of sulfur dichloride with allyl thymol 5 and allyl carvacrol 6 was very sluggish under the same conditions as synthesis of selenium analogues $\mathbf{7}$ and $\mathbf{8}$. Heating under reflux in chloroform was found to be necessary in order to accelerate the reaction. Besides, using a $20 \%$ excess of sulfur dichloride is advisable, otherwise a part of allyl thymol 5 and allyl carvacrol 6 stayed unconverted. After heating under reflux, sodium hydrocarbonate was added in order to catalyze intramolecular nucleophilic substitution and the reaction mixture was stirred overnight at room temperature. Bis[(7-isopropyl-4methyl-2,3-dihydro-1-benzofuran-2-yl)methyl] and bis[(4-isopropyl-7-methyl-2,3-dihydro1-benzofuran-2-yl)methyl] sulfides 9 and 10 were isolated by column chromatography on silica gel in 70 and $72 \%$ yields, respectively (Scheme 4).<smiles>C=CCc1c(C)ccc(C(C)C)c1O</smiles>

5<smiles>C=CCc1c(C(C)C)ccc(C)c1O</smiles>
6

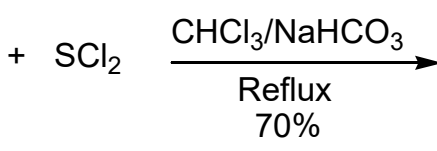

$+\mathrm{SCl}_{2}$

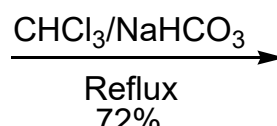<smiles>Cc1ccc(C(C)C)c2c1CC(CSCC1Cc3c(C)ccc(C(C)C)c3O1)O2</smiles>

9<smiles>Cc1ccc(C(C)C)c2c1CC(CSCC1Cc3c(C(C)C)ccc(C)c3O1)O2</smiles>

10

Scheme 4. Synthesis of sulfides 9 and $\mathbf{1 0}$ by the reaction of sulfur dichloride with allyl thymol 5 and allyl carvacrol 6.

We suppose that heating accelerates isomerization of primarily formed anti-Markovnikov adducts A into Markovnikov products B followed by intramolecular nucleophilic substitution with the formation of (2,3-dihydro-1-benzofuran-2-yl)methyl moiety (Scheme 5). It is known that electrophilic addition of sulfenyl halides to 1-alkenes leads predominantly to anti-Markovnikov products [73-75]. 
<smiles>[X]CC(Cc1c([AlH])ccc([R])c1O)Cc1c([AlH2])ccc([R])c1O</smiles>

5,6

A

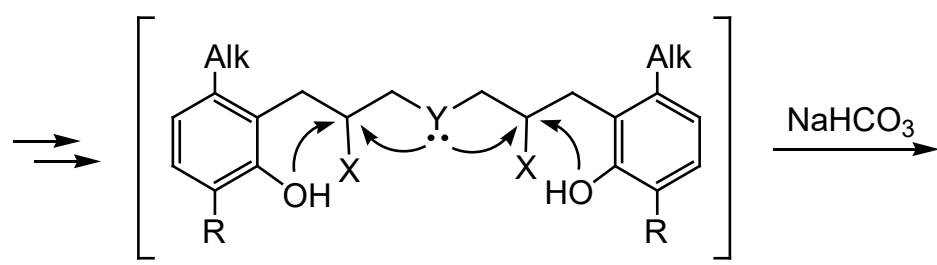

B<smiles>[Y]CC1Cc2c([AlH2])ccc([R])c2O1</smiles>

7-10

$\mathrm{YX}_{2}=\mathrm{SeBr}_{2}, \mathrm{SeCl}_{2}(\mathbf{7}, \mathbf{8}) ; \mathrm{YX}_{2}=\mathrm{SCl}_{2}(\mathbf{9}, \mathbf{1 0}) ; \mathrm{Alk}=\mathrm{Me}, \mathrm{R}=\mathrm{i}-\mathrm{Pr}(\mathbf{5}, \mathbf{7}, \mathbf{9}) ; \mathrm{Alk}=\mathrm{i}-\mathrm{Pr}, \mathrm{R}=\mathrm{Me}(\mathbf{6}, \mathbf{8}, \mathbf{1 0})$

Scheme 5. A supposed pathway of the reactions of $\mathrm{SeBr}_{2}, \mathrm{SeCl}_{2}$ and $\mathrm{SCl}_{2}$ with allyl thymol 5 and allyl carvacrol 6.

We suppose that similar consequence of transformations (Scheme 5) also takes place in selenocyclofunctionalization reactions (Scheme 3). The isomerization process and intramolecular nucleophilic substitution proceed smoothly at room temperature due to high anchimeric assistance effect of the selenium atom, which activates halogen atoms in $\beta$-haloorganyl selenides B (Scheme 5) [58]. Previously, we observed the formation of anti-Markovnikov adducts in the reactions of selenium dibromide with 1-alkenes and their isomerization into thermodynamically more stable Markovnikov products at room temperature [76,77]. The bromine atom is better leaving group compared to the chlorine atom in nucleophilic substitution and selenium dibromide is more efficient than selenium dichloride in these reactions.

Tellurium tetrachloride and tetrabromide were used in the cyclofunctionalization reactions with allyl thymol 5 and allyl carvacrol $\mathbf{6}$, and conditions for efficient selective reactions with each of these tellurium-centered electrophilic reagents were established. We found that the reactions of tellurium tetrachloride with allyl thymol 5 and allyl carvacrol 6 proceeded smoothly in methylene chloride at the temperature $35-40{ }^{\circ} \mathrm{C}$ affording trichlorotellanes 11 and 12 bearing the (2,3-dihydro-1-benzofuran-2-yl)methyl moiety in quantitative yields (Scheme 6).<smiles>C=CCc1c(C)ccc(C(C)C)c1O</smiles>

5<smiles>C=CCc1c(C(C)C)ccc(C)c1O</smiles>

6<smiles>Cc1ccc(C(C)C)c2c1CC(C[Te]Cl)O2</smiles>

11<smiles>Cc1ccc(C(C)C)c2c1OC(C[Te]Cl)C2</smiles>

12

Scheme 6. Synthesis of tellanes $\mathbf{1 1}$ and $\mathbf{1 2}$ by the reaction of tellurium tetrachloride with allyl thymol 5 and allyl carvacrol 6. 
The disadvantage of using tellurium tetrabromide is the low solubility of this reagent in most common organic solvents (chloroform, methylene chloride, benzene, diethyl ether, carbon tetrachloride). However, we found that the reactions of tellurium tetrabromide with allyl thymol 5 and allyl carvacrol 6 can be efficiently carried out in acetonitrile at room temperature affording tribromotellanes $\mathbf{1 3}$ and $\mathbf{1 4}$ in quantitative yield (Scheme 7).<smiles>C=CCc1c(C)ccc(C(C)C)c1O</smiles>

5<smiles>C=CCc1c(C(C)C)ccc(C)c1O</smiles>

6<smiles>Cc1ccc(C(C)C)c2c1CC(C[Te](C)(C)C)O2</smiles>

13<smiles>Cc1ccc(C(C)C)c2c1OC(C[Te](C)(C)C)C2</smiles>

14

Scheme 7. Synthesis of tellanes $\mathbf{1 3}$ and $\mathbf{1 4}$ by the reaction of tellurium tetrabromide with allyl thymol 5 and carvacrol 6.

The intramolecular substitution is activated by anchimeric assistance of the selenium atom in the reactions with selenium dibromide (Scheme 3), however, in the case of tellurium tetrachloride and tetrabromide (Schemes 6 and 7), the halogen atom in the addition products is very reactive due to high electron-withdrawing effect of trichlorotellanyl and tribromotellanyl groups.

It is worth noting that reactions of tellurium tetrachloride and tetrabromide with allyl thymol 5 and allyl carvacrol $\mathbf{6}$ proceeded with high selectivity in a regiospecific manner affording only monoadducts 11-14 in quantitative yields (Schemes 6 and 7).

Finally, we obtained functionalized ditellurides 15 and 16 bearing the (2,3-dihydro-1benzofuran-2-yl)methyl moiety in 91-92\% yields by the reduction of trichlorotellanes $\mathbf{1 1}$ and 12 with sodium metabisulfite (Scheme 8). The reactions were selectively carried out in the two-phase solvent system of water and methylene chloride at room temperature giving the products 15 and 16, which did not require additional purification.<smiles>Cc1ccc(C(C)C)c2c1CC(C[Te]Cl)O2</smiles>

11<smiles>Cc1ccc(C(C)C)c2c1OC(C[Te]Cl)C2</smiles>

12
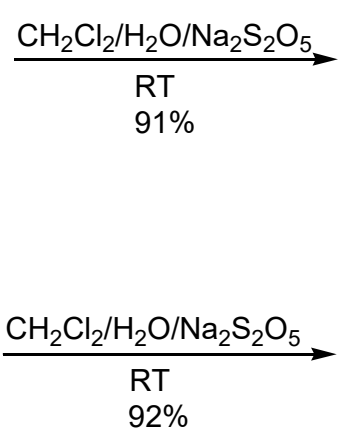

$92 \%$

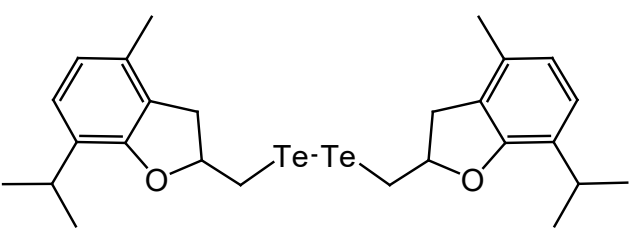

15

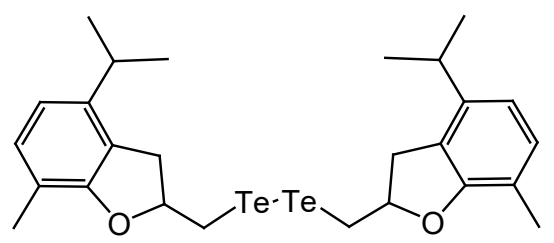

16

Scheme 8. Synthesis of ditellurides $\mathbf{1 5}$ and $\mathbf{1 6}$ by the reduction of trichlorotellanes $\mathbf{1 1}$ and $\mathbf{1 2}$ with sodium metabisulfite. 
The obtained products represent a new family of organochalcogen compounds bearing (7-isopropyl-4-methyl-2,3-dihydro-1-benzofuran-2-yl)methyl and (4-isopropyl-7-methyl2,3-dihydro-1-benzofuran-2-yl)methyl moieties with promising biological activity.

The structural assignments of the synthesized compounds were made using ${ }^{1} \mathrm{H}-,{ }^{13} \mathrm{C}-$, ${ }^{77} \mathrm{Se}-$ and ${ }^{125} \mathrm{Te}-\mathrm{NMR}$ spectroscopy and confirmed by elemental analysis. The compounds $\mathbf{7 - 1 0}, \mathbf{1 5}, 16$ consist of two diastereomers ( $d l$ and meso) approximately in an equimolar ratio. Two closely spaced signals which correspond to two diastereomers were observed in the ${ }^{77}$ Se NMR spectra of compounds 7 and 8.

The signals of the $\mathrm{CH}_{2} \mathrm{TeCl}_{3}$ and $\mathrm{CH}_{2} \mathrm{TeBr}_{3}$ groups in ${ }^{13} \mathrm{C}-\mathrm{NMR}$ spectra of compounds 11-14 manifest themselves in the downfield region (66.0-66.3 and $~ 61.5 \mathrm{ppm}$ ) due to high electron-withdrawing effect of trichlorotellanyl and tribromotellanyl groups. The signals of the $\mathrm{CH}_{2}$ Te group in ${ }^{13} \mathrm{C}-\mathrm{NMR}$ spectra of ditellurides $\mathbf{1 5}$ and $\mathbf{1 6}$ are observed in the upfield region ( $11.8 \mathrm{ppm})$.

The tellurium atom of the $\mathrm{CH}_{2} \mathrm{TeCl}_{3}$ group in ${ }^{125} \mathrm{Te} \mathrm{NMR}$ spectra of compounds 11 and 12 resonated in the downfield region (1206.3 and $1204.0 \mathrm{ppm}$ ) due to the presence of three electronegative chlorine atoms. However, the signals in ${ }^{125} \mathrm{Te}-\mathrm{NMR}$ spectra of ditellurides 15 and 16, which were obtained by the reduction of compounds 11 and 12, were observed in the upfield region (77.9 and $84.0 \mathrm{ppm})$.

\section{Experimental Section}

\subsection{General Information}

The ${ }^{1} \mathrm{H}(400.1 \mathrm{MHz}),{ }^{13} \mathrm{C}(100.6 \mathrm{MHz}),{ }^{77} \mathrm{Se}(76.3 \mathrm{MHz})$ and ${ }^{125} \mathrm{Te}(126.4 \mathrm{MHz}) \mathrm{NMR}$ spectra (see Supplementary Materials) were recorded on a Bruker DPX-400 spectrometer (Bruker BioSpin $\mathrm{GmbH}$, Rheinstetten, Germany) in $\mathrm{CDCl}_{3}$ and $d_{6}$-DMSO solutions and referred to the residual solvent peaks of $\mathrm{CDCl}_{3}\left(\delta=7.27\right.$ and $77.16 \mathrm{ppm}$ in ${ }^{1} \mathrm{H}$ - and ${ }^{13} \mathrm{C}$ $\mathrm{NMR}$, respectively) and $d_{6}$-DMSO ( $\delta=2.50$ and $39.5 \mathrm{ppm}$ in ${ }^{1} \mathrm{H}$ - and $\left.{ }^{13} \mathrm{C}-\mathrm{NMR}\right)$ or $\mathrm{Me}_{2} \mathrm{Se}$ ( ${ }^{77} \mathrm{Se}-\mathrm{NMR}$, external) and $\mathrm{Me}_{2} \mathrm{Te}\left({ }^{125} \mathrm{Te}-\mathrm{NMR}\right.$, external). Elemental analysis was performed on a Thermo Scientific Flash 2000 Elemental Analyzer (Thermo Fisher Scientific Inc., Milan, Italy). Melting points were determined on a Kofler Hot-Stage Microscope PolyTherm A apparatus (Wagner \& Munz GmbH, Munich, Germany). The organic solvents were dried and distilled according to standard procedures. Silica gel (0.06-0.20 mm (70-230 mesh, Alfa Aesar, Heysham, Lancashire, United Kingdom) was used for column chromatography.

\subsection{Synthesis of Starting Compounds $\mathbf{5}$ and $\mathbf{6}$}

2-Allyl-6-isopropyl-3-methylphenol (5). A mixture of allyl thymyl ether 3 (4 g, $21 \mathrm{mmol})$ and dry powdered $\mathrm{NaHCO}_{3}(1 \mathrm{~g}, 12 \mathrm{mmol})$ was heated at $200{ }^{\circ} \mathrm{C}$ for $2 \mathrm{~h}$ in a sealed tube under argon. Compound 5 (2.84 g, 71\% yield) was isolated as a colourless liquid by column chromatography on silica gel (eluent: hexane, then hexane/methylene chloride 1:10).

${ }^{1} \mathrm{H}-\mathrm{NMR}\left(\mathrm{CDCl}_{3}\right): 1.39\left(\mathrm{~d}, 6 \mathrm{H}, \mathrm{CH}_{3} \mathrm{CH}, J=7 \mathrm{~Hz}\right), 2.41\left(\mathrm{~s}, 3 \mathrm{H}, \mathrm{CH}_{3} \mathrm{C}_{\mathrm{Ar}}\right), 3.26-3.35(\mathrm{~m}$, $\left.1 \mathrm{H}, \mathrm{CH}_{3} \mathrm{C} \underline{\mathrm{H}}\right), 3.55-3.58\left(\mathrm{~m}, 2 \mathrm{H}, \mathrm{CH}_{2} \mathrm{C}_{\mathrm{Ar}}\right), 5.10(\mathrm{~s}, 1 \mathrm{H}, \mathrm{OH}), 5.21-5.27\left(\mathrm{~m}, 2 \mathrm{H}, \mathrm{CH}_{2}=\mathrm{CH}\right)$, 6.06-6.16 (m, 1H, $\left.\mathrm{CH}_{2}=\mathrm{C} \underline{\mathrm{H}}\right), 6.91\left(\mathrm{~d}, 1 \mathrm{H}, \mathrm{CH}_{\mathrm{Ar}}, J=7.8 \mathrm{~Hz}\right), 7.15\left(\mathrm{~d}, 1 \mathrm{H}, \mathrm{CH}_{\mathrm{Ar}}, J=7.8 \mathrm{~Hz}\right)$. ${ }^{13} \mathrm{C}-\mathrm{NMR}\left(\mathrm{CDCl}_{3}\right): 19.7\left(\underline{\mathrm{CH}}_{3} \mathrm{C}_{\mathrm{Ar}}\right), 22.8\left(\underline{\mathrm{CH}}{ }_{3} \mathrm{CH}\right), 27.0\left(\mathrm{CH}_{3} \underline{\mathrm{CH}}\right), 31.3\left(\underline{\mathrm{CH}}_{2} \mathrm{C}_{\mathrm{Ar}}\right), 116.0$ $\left(\mathrm{CH}_{2}=\mathrm{CH}\right), 122.5\left(\mathrm{CH}_{\mathrm{Ar}}\right), 123.2\left(\mathrm{CH}_{2} \underline{\mathrm{C}}_{\mathrm{Ar}}\right), 123.9\left(\mathrm{CH}_{\mathrm{Ar}}\right), 132.4\left(\mathrm{CHC}_{\mathrm{Ar}}\right), 134.9\left(\mathrm{CH}_{3} \underline{\mathrm{C}}_{\mathrm{Ar}}\right)$, $135.7\left(\mathrm{CH}_{2}=\underline{\mathrm{CH}}\right), 151.6\left(\mathrm{C}_{\mathrm{Ar}} \mathrm{OH}\right)$. Anal. calcd for $\mathrm{C}_{13} \mathrm{H}_{18} \mathrm{O}$ (190.28): C 82.06, $\mathrm{H} 9.53 \%$. Found: C 82.31, H 9.38\%.

2-Allyl-3-isopropyl-6-methylphenol (6) was obtained as a colourless liquid $(2.80 \mathrm{~g}, 70 \%$ yield) from allyl carvacryl ether $4(4 \mathrm{~g}, 21 \mathrm{mmol})$ and dry powdered $\mathrm{NaHCO}_{3}(1 \mathrm{~g}, 12 \mathrm{mmol})$ by heating the mixture at $200{ }^{\circ} \mathrm{C}$ for $2 \mathrm{~h}$ in a sealed tube followed by isolation by column chromatography on silica gel (eluent: hexane, then hexane/methylene chloride 1:10). ${ }^{1} \mathrm{H}-\mathrm{NMR}\left(\mathrm{CDCl}_{3}\right): 1.34\left(\mathrm{~d}, 6 \mathrm{H}, \mathrm{CH}_{3} \mathrm{CH}, J=7 \mathrm{~Hz}\right), 2.34\left(\mathrm{~s}, 3 \mathrm{H}, \mathrm{CH}_{3} \mathrm{C}_{\mathrm{Ar}}\right), 3.19-3.25(\mathrm{~m}$, $\left.1 \mathrm{H}, \mathrm{CH}_{3} \underline{\mathrm{H}}\right), 3.60-3.63\left(\mathrm{~m}, 2 \mathrm{H}, \mathrm{CH}_{2} \mathrm{C}_{\mathrm{Ar}}\right), 5.03(\mathrm{~s}, 1 \mathrm{H}, \mathrm{OH}), 5.16-5.25\left(\mathrm{~m}, 2 \mathrm{H}, \mathrm{CH}_{2}=\mathrm{CH}\right)$, 6.08-6.18 (m, 1H, $\left.\mathrm{CH}_{2}=\mathrm{C} \underline{\mathrm{H}}\right), 6.95\left(\mathrm{~d}, 1 \mathrm{H}, \mathrm{CH}_{\mathrm{Ar}}, J=7.9 \mathrm{~Hz}\right), 7.14\left(\mathrm{~d}, 1 \mathrm{H}, \mathrm{CH}_{\mathrm{Ar}}, J=7.9 \mathrm{~Hz}\right)$ ${ }^{13} \mathrm{C}-\mathrm{NMR}\left(\mathrm{CDCl}_{3}\right): 15.9\left(\mathrm{CH}_{3} \mathrm{C}_{\mathrm{Ar}}\right) 24.1\left(\mathrm{CH}_{3} \mathrm{CH}\right), 29.3\left(\mathrm{CH}_{3} \underline{\mathrm{CH}}\right), 30.1\left(\underline{\mathrm{CH}}_{2} \mathrm{C}_{\mathrm{Ar}}\right), 115.8$ $\left(\underline{\mathrm{CH}}_{2}=\mathrm{CH}\right), 117.3\left(\mathrm{CH}_{\mathrm{Ar}}\right), 121.1\left(\mathrm{CH}_{3} \underline{\mathrm{C}}_{\mathrm{Ar}}\right), 122.1\left(\mathrm{CH}_{2} \underline{\mathrm{C}}_{\mathrm{Ar}}\right), 128.9\left(\mathrm{CH}_{\mathrm{Ar}}\right), 136.6\left(\mathrm{CH}_{2}=\underline{\mathrm{CH}}\right)$, 
146.1 ( $\left.\mathrm{CHC}_{\mathrm{Ar}}\right), 152.4\left(\mathrm{C}_{\mathrm{Ar}} \mathrm{OH}\right)$. Anal. calcd for $\mathrm{C}_{13} \mathrm{H}_{18} \mathrm{O}$ (190.28): $\mathrm{C} 82.06, \mathrm{H} 9.53 \%$. Found: C $82.24, \mathrm{H} 9.67 \%$.

\subsection{Synthesis of Products $\mathbf{7 - 1 6}$}

Bis[(7-isopropyl-4-methyl-2,3-dihydro-1-benzofuran-2-yl)methyl] selenide (7). A solution of selenium dibromide $(0.5 \mathrm{mmol})$ was prepared from selenium $(0.04 \mathrm{~g}, 0.5 \mathrm{mmol})$ and bromine $(0.08 \mathrm{~g}, 0.5 \mathrm{mmol})$ in methylene chloride $(2 \mathrm{~mL})$. The obtained solution of selenium dibromide $(0.5 \mathrm{mmol})$ was added dropwise to a solution of allyl thymol $5(0.19 \mathrm{~g}, 1 \mathrm{mmol})$ in methylene chloride $(10 \mathrm{~mL})$. The mixture was stirred for $7 \mathrm{~h}$ at room temperature and $\mathrm{NaHCO}_{3}(0.17 \mathrm{~g}, 2 \mathrm{mmol})$ was added. The mixture was stirred overnight $(14 \mathrm{~h})$ at room temperature. The mixture was filtered and the solvent was removed by a rotary evaporator. The residue was subjected to column chromatography on silica gel (eluent: hexane, then hexane/chloroform 1:10). Compound $7(0.206 \mathrm{~g}, 90 \%$ yield $)$ was isolated as a colourless viscous oil. ${ }^{1} \mathrm{H}-\mathrm{NMR}\left(\mathrm{CDCl}_{3}\right): 1.26\left(\mathrm{~d}, 12 \mathrm{H}, \mathrm{CH}_{3} \mathrm{CH}, J=6.8 \mathrm{~Hz}\right), 2.19(\mathrm{~d}$, $\left.6 \mathrm{H}, \mathrm{CH}_{3} \mathrm{C}_{\mathrm{Ar}}, J=7.2 \mathrm{~Hz}\right), 2.83-2.91\left(\mathrm{~m}, 2 \mathrm{H}, \mathrm{CH}_{3} \mathrm{C} \underline{\mathrm{H}}\right), 2.93-3.06\left(\mathrm{~m}, 4 \mathrm{H}, \mathrm{CH}_{2} \mathrm{Se}, \mathrm{CH}_{2} \mathrm{C}_{\mathrm{Ar}}\right)$, 3.10-3.20 (m, 2H, $\left.\underline{\mathrm{H}}_{2} \mathrm{Se}\right), 3.35-3.43\left(\mathrm{~m}, 2 \mathrm{H}, \mathrm{CH}_{2} \mathrm{C}_{\mathrm{Ar}}\right), 5.02-5.09(\mathrm{~m}, 2 \mathrm{H}, \mathrm{CHO}), 6.71(\mathrm{~d}$, $\left.2 \mathrm{H}, \mathrm{CH}_{\mathrm{Ar}}, J=7.7 \mathrm{~Hz}\right), 6.95\left(\mathrm{~d}, 2 \mathrm{H}, \mathrm{CH}_{\mathrm{Ar}}, J=7.7 \mathrm{~Hz}\right) .{ }^{13} \mathrm{C}-\mathrm{NMR}\left(\mathrm{CDCl}_{3}\right): 15.1\left(\mathrm{CH}_{3} \mathrm{C}_{\mathrm{Ar}}\right)$, $15.1\left(\mathrm{CH}_{3} \mathrm{C}_{\mathrm{Ar}}\right), 23.0\left(\mathrm{CH}_{3} \mathrm{CH}\right), 23.0\left(\mathrm{CH}_{3} \mathrm{CH}\right), 29.7\left(\mathrm{CH}_{2} \mathrm{Se}\right), 30.0\left(\mathrm{CH}_{2} \mathrm{Se}\right), 31.4\left(\mathrm{CH}_{3} \mathrm{CH}\right)$, $35.0\left(\mathrm{CH}_{2} \mathrm{C}_{\mathrm{Ar}}\right), 82.5(\mathrm{CHO}), 117.0\left(\mathrm{CH}_{\mathrm{Ar}}\right), 123.2\left(\mathrm{CH}_{2} \mathrm{C}_{\mathrm{Ar}}\right), 123.2\left(\mathrm{CH}_{3} \mathrm{C}_{\mathrm{Ar}}\right), 129.7\left(\mathrm{CH}_{\mathrm{Ar}}\right)$, 143.0 ( $\left(\mathrm{CHC}_{\mathrm{Ar}}\right), 157.4\left(\mathrm{C}_{\mathrm{Ar}} \mathrm{O}\right) .{ }^{77} \mathrm{Se}-\mathrm{NMR}\left(\mathrm{CDCl}_{3}\right)$ : 103.1, 104.4. Anal. calcd for $\mathrm{C}_{26} \mathrm{H}_{34} \mathrm{O}_{2} \mathrm{Se}$ (457.51): C 68.26, H 7.49, Se 17.26\%. Found: C 68.48, H 7.64, Se 17.49\%.

Bis[(4-isopropyl-7-methyl-2,3-dihydro-1-benzofuran-2-yl)methyl] selenide (8) was obtained as a colourless viscous oil $(0.211 \mathrm{~g}, 92 \%$ from selenium dibromide $(0.5 \mathrm{mmol})$ and allyl carvacrol $6(0.19 \mathrm{~g}, 1 \mathrm{mmol})$ in methylene chloride under the same conditions as compound 7. ${ }^{1} \mathrm{H}-\mathrm{NMR}\left(\mathrm{CDCl}_{3}\right): 1.27\left(\mathrm{~d}, 12 \mathrm{H}, \mathrm{CH}_{3} \mathrm{CH}, J=6.8 \mathrm{~Hz}\right), 2.25\left(\mathrm{~s}, 6 \mathrm{H}, \mathrm{CH}_{3} \mathrm{C}_{\mathrm{Ar}}\right), 2.94-3.02$ $\left(\mathrm{m}, 4 \mathrm{H}, \mathrm{CH}_{3} \mathrm{CH}, \mathrm{CH}_{2} \mathrm{Se}\right), 3.06-3.19\left(\mathrm{~m}, 4 \mathrm{H}, \mathrm{CH}_{2} \mathrm{Se}, \mathrm{CH}_{2} \mathrm{C}_{\mathrm{Ar}}\right), 3.29-3.36\left(\mathrm{~m}, 2 \mathrm{H}, \mathrm{CH}_{2} \mathrm{C}_{\mathrm{Ar}}\right)$, $5.05-5.09(\mathrm{~m}, \overline{2 \mathrm{H}}, \mathrm{CHO}), 6.69\left(\mathrm{~d}, 2 \mathrm{H}, \mathrm{CH}_{\mathrm{Ar}}, J=7.6 \mathrm{~Hz}\right), 6.97\left(\mathrm{~d}, 2 \mathrm{H}, \mathrm{CH}_{\mathrm{Ar}}, J=7.6 \mathrm{~Hz}\right)$. ${ }^{13} \mathrm{C}-\mathrm{NMR}\left(100 \mathrm{MHz}, \mathrm{CDCl}_{3}\right)$ : $18.7\left(\mathrm{CH}_{3} \mathrm{Ar}\right), 22.6\left(\mathrm{CH}_{3} \mathrm{CH}\right), 28.2\left(\mathrm{CH}_{3} \mathrm{CH}\right), 29.7\left(\mathrm{CH}_{2} \mathrm{Se}\right)$, $30.0\left(\mathrm{CH}_{2} \mathrm{Se}\right), 35.1\left(\mathrm{CH}_{2} \mathrm{Ar}\right), 82.5(\mathrm{CHO}), 121.6\left(\mathrm{HC}_{\mathrm{Ar}}\right), 124.9\left(\mathrm{CH}_{2} \mathrm{C}_{\mathrm{Ar}}\right), 125.1\left(\mathrm{HC}_{\mathrm{Ar}}\right), 127.9$ $\left(\mathrm{CHC}_{\mathrm{Ar}}\right), 132.0\left(\mathrm{CH}_{3} \underline{\mathrm{C}}_{\mathrm{Ar}}\right), 156.4\left(\mathrm{OC}_{\mathrm{Ar}}\right) .{ }^{77} \mathrm{Se} \mathrm{NMR}\left(, \mathrm{CDCl}_{3}\right): 106.4,108.2$. Anal. calcd for $\mathrm{C}_{26} \mathrm{H}_{34} \mathrm{O}_{2} \mathrm{Se}$ (457.51): $\mathrm{C}$ 68.26, H 7.49, Se 17.26\%. Found: C 67.98, H 7.65, Se $17.47 \%$.

Bis[(7-isopropyl-4-methyl-2,3-dihydro-1-benzofuran-2-yl)methyl] sulfide (9). A solution of sulfur dichloride $(0.62 \mathrm{~g}, 0.6 \mathrm{mmol})$ in methylene chloride $(2 \mathrm{~mL})$ was added dropwise to a solution of allyl thymol $5(0.19 \mathrm{~g}, 1 \mathrm{mmol})$ in methylene chloride $(10 \mathrm{~mL})$. The mixture was stirred for $1 \mathrm{~h}$ at room temperature and refluxed for $7 \mathrm{~h} . \mathrm{NaHCO}_{3}(0.25 \mathrm{~g}, 3 \mathrm{mmol})$ was added and the mixture was stirred overnight $(14 \mathrm{~h})$ at room temperature. The mixture was filtered and the solvent was removed by a rotary evaporator. The residue was subjected to column chromatography on silica gel (eluent: hexane, then hexane/chloroform 1:10). Compound $9(0.144 \mathrm{~g}, 70 \%$ yield $)$ was isolated as a colourless viscous oil. ${ }^{1} \mathrm{H}-\mathrm{NMR}$ $\left(\mathrm{CDCl}_{3}\right): 1.25\left(\mathrm{~d}, 12 \mathrm{H}, \mathrm{CH}_{3} \mathrm{CH}, J=6.6 \mathrm{~Hz}\right), 2.24\left(\mathrm{~s}, 6 \mathrm{H}, \mathrm{CH}_{3} \mathrm{C}_{\mathrm{Ar}}\right), 2.88-2.96\left(\mathrm{~m}, 2 \mathrm{H}, \mathrm{CH}_{3} \mathrm{C} \underline{\mathrm{H}}\right)$, 2.97-3.02 (m, 2H, $\left.\mathrm{CH}_{2} \mathrm{~S}\right), 3.05-3.08\left(\mathrm{~m}, 2 \mathrm{H}, \mathrm{CH}_{2} \mathrm{C}_{\mathrm{Ar}}\right), 3.09-3.16\left(\mathrm{~m}, 2 \mathrm{H}, \mathrm{CH}_{2} \mathrm{~S}\right), 3.25-3.31$ $\left(\mathrm{m}, 2 \mathrm{H}, \mathrm{CH}_{2} \mathrm{C}_{\mathrm{Ar}}\right), 4.98-5.06(\mathrm{~m}, 2 \mathrm{H}, \mathrm{CHO}), 6.68\left(\mathrm{~d}, 2 \mathrm{H}, \mathrm{CH}_{\mathrm{Ar}}, J=7.9 \mathrm{~Hz}\right), 6.96(\mathrm{~d}, 2 \mathrm{H}$, $\left.\mathrm{CH}_{\mathrm{Ar}}, J=7.9 \mathrm{~Hz}\right) .{ }^{13} \mathrm{C}-\mathrm{NMR}\left(\mathrm{CDCl}_{3}\right): 18.7\left(\mathrm{CH}_{3} \mathrm{C}_{\mathrm{Ar}}\right), 22.6\left(\mathrm{CH}_{3} \mathrm{CH}\right), 28.2\left(\mathrm{CH}_{3} \underline{\mathrm{CH}}\right), 34.3$ $\left(\mathrm{CH}_{2} \mathrm{C}_{\mathrm{Ar}}\right), 37.7\left(\mathrm{CH}_{2} \mathrm{~S}\right), 38.1\left(\mathrm{CH}_{2} \mathrm{~S}\right), 82.1(\mathrm{CHO}), 121.6\left(\mathrm{CH}_{\mathrm{Ar}}\right), 124.8\left(\mathrm{CH}_{2} \underline{\mathrm{C}}_{\mathrm{Ar}}\right), 125.1$ $\left(\mathrm{CH}_{\mathrm{Ar}}\right), 127.9\left(\mathrm{CHC}_{\mathrm{Ar}}\right), 132.1\left(\mathrm{CH}_{3} \underline{\mathrm{C}}_{\mathrm{Ar}}\right), 156.4\left(\mathrm{C}_{\mathrm{Ar}} \mathrm{O}\right)$. Anal. calcd for $\mathrm{C}_{26} \mathrm{H}_{34} \mathrm{O}_{2} \mathrm{~S}(410.61)$ : C 76.05, H 8.35, S 7.81\%. Found: C 76.28, H 8.19, S 7.65\%.

Bis[(4-isopropyl-7-methyl-2,3-dihydro-1-benzofuran-2-yl)methyl] sulfide (10) was obtained as a colourless viscous oil ( $0.148 \mathrm{~g}, 72 \%$ yield) from sulfur dichloride $(0.62 \mathrm{~g}, 0.6 \mathrm{mmol})$ and allyl carvacrol $6(0.19 \mathrm{~g}, 1 \mathrm{mmol})$ in methylene chloride under the same conditions as compound 9. ${ }^{1} \mathrm{H}-\mathrm{NMR}\left(\mathrm{CDCl}_{3}\right): 1.23\left(\mathrm{~d}, 12 \mathrm{H}, \mathrm{CH}_{3} \mathrm{CH}, J=6.5 \mathrm{~Hz}\right), 2.16\left(\mathrm{~d}, 6 \mathrm{H}, \mathrm{CH}_{3} \mathrm{C}_{\mathrm{Ar}}\right.$, $J=10.8 \mathrm{~Hz}), 2.81-2.95\left(\mathrm{~m}, 4 \mathrm{H}, \mathrm{CH}_{3} \mathrm{CH}, \mathrm{CH}_{2} \mathrm{~S}\right), 2.99-3.06\left(\mathrm{~m}, 2 \mathrm{H}, \mathrm{CH}_{2} \mathrm{C}_{\mathrm{Ar}}\right), 3.09-3.15(\mathrm{~m}, 2 \mathrm{H}$, $\left.\mathrm{CH}_{2} \mathrm{~S}\right), 3.30-3.36\left(\mathrm{~m}, 2 \mathrm{H}, \mathrm{CH}_{2} \mathrm{C}_{\mathrm{Ar}}\right), 4.95-5.02(\mathrm{~m}, 2 \mathrm{H}, \mathrm{CHO}), 6.68\left(\mathrm{~d}, 2 \mathrm{H}, \mathrm{CH}_{\mathrm{Ar}}, J=7.5 \mathrm{~Hz}\right)$, $6.91\left(\mathrm{~d}, 2 \mathrm{H}, \mathrm{CH}_{\mathrm{Ar}}, J=7.5 \mathrm{~Hz}\right) .{ }^{13} \mathrm{C}-\mathrm{NMR}\left(\mathrm{CDCl}_{3}\right): 15.1\left(\mathrm{CH}_{3} \mathrm{C}_{\mathrm{Ar}}\right), 15.1\left(\mathrm{CH}_{3} \mathrm{C}_{\mathrm{Ar}}\right), 23.0$ $\left(\underline{\mathrm{C}} \mathrm{H}_{3} \mathrm{CH}\right), 23.0\left(\underline{\mathrm{C}} \mathrm{H}_{3} \mathrm{CH}\right), 31.5\left(\underline{\mathrm{CH}}_{2} \mathrm{C}_{\mathrm{Ar}}\right), 34.3\left(\mathrm{CH}_{3} \underline{\mathrm{CH}}\right), 37.8\left(\mathrm{CH}_{2} \mathrm{~S}\right), 38.2\left(\underline{\mathrm{C}} \mathrm{H}_{2} \mathrm{~S}\right), 82.3$ 
(CHO), $117.0\left(\mathrm{CH}_{3} \underline{\mathrm{C}}_{\mathrm{Ar}}\right), 117.1\left(\mathrm{CH}_{\mathrm{Ar}}\right), 123.2\left(\mathrm{CH}_{2} \underline{\mathrm{C}}_{\mathrm{Ar}}\right), 129.7\left(\mathrm{CH}_{\mathrm{Ar}}\right), 143.0(\mathrm{CHC} \underline{\mathrm{Ar}}), 157.4$ $\left(\mathrm{C}_{\mathrm{Ar}} \mathrm{O}\right)$. Anal. calcd for $\mathrm{C}_{26} \mathrm{H}_{34} \mathrm{O}_{2} \mathrm{~S}$ (410.61): C 76.05, H 8.35, S 7.81\%. Found: C 75.91, H 8.54, S $7.76 \%$.

Trichloro[(7-isopropyl-4-methyl-2,3-dihydro-1-benzofuran-2-yl)methyl]- $\lambda^{4}$-tellane (11). A solution of allyl thymol $5(0.19 \mathrm{~g}, 1 \mathrm{mmol})$ in methylene chloride $(2 \mathrm{~mL})$ was added dropwise to a stirred mixture of tellurium tetrachloride $(0.27 \mathrm{~g}, 1 \mathrm{mmol})$ and methylene chloride $(10 \mathrm{~mL})$. The obtained mixture was stirred at room temperature for $2 \mathrm{~h}$ and at $35-40{ }^{\circ} \mathrm{C}$ for $6 \mathrm{~h}$. The solvent was removed by a rotary evaporator and the residue was dried in vacuum. Compound $11(0.423 \mathrm{~g}$, quantitative yield) was isolated as a grey viscous oil.

${ }^{1} \mathrm{H}-\mathrm{NMR}\left(\mathrm{CDCl}_{3}\right): 1.20\left(\mathrm{~d}, 3 \mathrm{H}, \mathrm{CH}_{3} \mathrm{CH}, J=7.1 \mathrm{~Hz}\right), 1.29\left(\mathrm{~d}, 3 \mathrm{H}, \mathrm{CH}_{3} \mathrm{CH}, J=7.1 \mathrm{~Hz}\right)$, 2.28 (s, 3H, $\left.\underline{\mathrm{H}}_{3} \mathrm{C}_{\mathrm{Ar}}\right), 2.97-3.04\left(\mathrm{~m}, 1 \mathrm{H}, \mathrm{CH}_{3} \mathrm{C} \underline{\mathrm{H}}\right), 3.26-3.33\left(\mathrm{~m}, 1 \mathrm{H}, \mathrm{CH}_{2} \mathrm{C}_{\mathrm{Ar}}\right.$ ), 3.51-3.57 $\left(\mathrm{m}, 1 \mathrm{H}, \mathrm{CH}_{2} \mathrm{C}_{\mathrm{Ar}}\right), 4.54-4.65\left(\mathrm{~m}, 2 \mathrm{H}, \mathrm{CH}_{2} \mathrm{Te}\right), 5.85-5.93(\mathrm{~m}, 1 \mathrm{H}, \mathrm{CHO}), 6.85\left(\mathrm{~d}, 1 \mathrm{H}, \mathrm{CH}_{\mathrm{Ar}}\right.$, $J=7.7 \mathrm{~Hz}), 7.08\left(\mathrm{~d}, 1 \mathrm{H}, \mathrm{CH}_{\mathrm{Ar}}, J=7.7 \mathrm{~Hz}\right) .{ }^{13} \mathrm{C}-\mathrm{NMR}\left(\mathrm{DMSO}-d_{6}\right): 18.5\left(\mathrm{CH}_{3} \mathrm{C}_{\mathrm{Ar}}\right), 22.4$ $\left(\mathrm{CH}_{3} \mathrm{CH}\right), 22.5\left(\mathrm{CH}_{3} \mathrm{CH}\right), 27.8\left(\mathrm{CH}_{3} \mathrm{CH}\right), 35.3\left(\mathrm{CH}_{2} \mathrm{C}_{\mathrm{Ar}}\right), 66.3\left(\mathrm{CH}_{2} \mathrm{Te}\right), 79.2(\mathrm{CHO}), 121.6$ $\left(\mathrm{CH}_{\mathrm{Ar}}\right), 124.7\left(\mathrm{CH}_{2} \underline{\mathrm{C}}_{\mathrm{Ar}}\right), 124.8\left(\mathrm{CH}_{\mathrm{Ar}}\right), 127.1\left(\mathrm{CH} \underline{\mathrm{C}}_{\mathrm{Ar}}\right), 131.7\left(\mathrm{CH}_{3} \underline{\mathrm{C}}_{\mathrm{Ar}}\right), 155.4(\mathrm{C} \mathrm{Ar}) .{ }^{125} \mathrm{Te}-$ NMR (DMSO-d $d_{6}$ ): 1206.3. Anal. calcd for $\mathrm{C}_{13} \mathrm{H}_{17} \mathrm{OCl}_{3} \mathrm{Te}$ (423.23): C 36.79, H 4.05, Cl 25.13, Te 30.15\%. Found: C 36.96, H 3.98, Cl 25.29, Te 29.98\%.

Trichloro[(4-isopropyl-7-methyl-2,3-dihydro-1-benzofuran-2-yl)methyl]- $\lambda^{4}$-tellane (12) was obtained as a colourless viscous oil ( $0.423 \mathrm{~g}$, quantitative yield) from tellurium tetrachloride $(0.27 \mathrm{~g}, 1 \mathrm{mmol})$ and allyl carvacrol $6(0.19 \mathrm{~g}, 1 \mathrm{mmol})$ in methylene chloride under the same conditions as compound 11. ${ }^{1} \mathrm{H}-\mathrm{NMR}\left(\mathrm{CDCl}_{3}\right): 1.20\left(\mathrm{~d}, 3 \mathrm{H}, \mathrm{C}_{3} \mathrm{CH}, J=6.9 \mathrm{~Hz}\right), 1.30$ $\left(\mathrm{d}, 3 \mathrm{H}, \mathrm{CH}_{3} \mathrm{CH}, J=6.9 \mathrm{~Hz}\right), 2.22\left(\mathrm{~s}, 3 \mathrm{H}, \mathrm{CH}_{3} \mathrm{C}_{\mathrm{Ar}}\right), 2.88-2.96\left(\mathrm{~m}, 1 \mathrm{H}, \mathrm{CH}_{3} \mathrm{CH}\right), 3.31-3.38$ $\left(\mathrm{m}, 1 \mathrm{H}, \mathrm{CH}_{2} \mathrm{C}_{\mathrm{Ar}}\right), 3.54-3.62\left(\mathrm{~m}, 1 \mathrm{H}, \mathrm{CH}_{2} \mathrm{C}_{\mathrm{Ar}}\right), 4.50-4.61\left(\mathrm{~m}, 2 \mathrm{H}, \mathrm{CH}_{2} \mathrm{Te}\right), 5.86-5.93(\mathrm{~m}, 1 \mathrm{H}$, $\mathrm{CHO}), 6.90\left(\mathrm{~d}, 1 \mathrm{H}, \mathrm{CH}_{\mathrm{Ar}}, J=7.8 \mathrm{~Hz}\right), 7.08\left(\mathrm{~d}, 1 \mathrm{H}, \mathrm{CH}_{\mathrm{Ar}}, J=7.8 \mathrm{~Hz}\right) .{ }^{13} \mathrm{C}-\mathrm{NMR}\left(\mathrm{DMSO}-d_{6}\right)$ : $15.0\left(\underline{\mathrm{CH}}_{3} \mathrm{C}_{\mathrm{Ar}}\right), 22.7\left(\underline{\mathrm{CH}}_{3} \mathrm{CH}\right), 23.0\left(\underline{\mathrm{CH}}{ }_{3} \mathrm{CH}\right), 30.0\left(\mathrm{CH}_{3} \underline{\mathrm{CH}}\right), 35.1\left(\underline{\mathrm{CH}}_{2} \mathrm{C}_{\mathrm{Ar}}\right), 66.0\left(\mathrm{CH}_{2} \mathrm{Te}\right)$, $79.2(\mathrm{CHO}), 116.2\left(\mathrm{CH}_{\mathrm{Ar}}\right), 117.1\left(\mathrm{CH}_{2} \mathrm{C}_{\mathrm{Ar}}\right), 122.9\left(\mathrm{CH}_{3} \underline{\mathrm{C}}_{\mathrm{Ar}}\right), 129.3\left(\mathrm{CH}_{\mathrm{Ar}}\right), 142.6\left(\mathrm{CHC}_{\mathrm{Ar}}\right)$, $156.4\left(\mathrm{C}_{\mathrm{Ar}} \mathrm{O}\right) .{ }^{125} \mathrm{Te}-\mathrm{NMR}$ (DMSO-d $)$ : 1204.0. Anal. calcd for $\mathrm{C}_{13} \mathrm{H}_{17} \mathrm{OCl}_{3} \mathrm{Te}$ (423.23): $\mathrm{C}$ 36.89, H 4.15, Cl 25.13, Te 30.15\%. Found: C 37.08, H 4.03, Cl 24.92, Te 30.32\%.

Tribromo[(7-isopropyl-4-methyl-2,3-dihydro-1-benzofuran-2-yl)methyl]- $\lambda^{4}$-tellane (13). A solution of allyl thymol $5(0.19 \mathrm{~g}, 1 \mathrm{mmol})$ in acetonitrile $(2 \mathrm{~mL})$ was added to a stirred mixture of tellurium tetrabromide $(0.27 \mathrm{~g}, 1 \mathrm{mmol})$ in acetonitrile $(8 \mathrm{~mL})$. The obtained mixture was stirred at room temperature overnight $(16 \mathrm{~h})$. The solvent was removed by a rotary evaporator and the residue was dried in vacuum. Compound 13 (0.423 g, quantitative yield) was obtained as a light orange powder, $\mathrm{mp} 61-63{ }^{\circ} \mathrm{C}$ (decomp.). ${ }^{1} \mathrm{H}$ $\operatorname{NMR}\left(\mathrm{CDCl}_{3}\right): 1.03\left(\mathrm{~d}, 3 \mathrm{H}, \mathrm{CH}_{3} \mathrm{CH}, J=6.8 \mathrm{~Hz}\right), 1.05\left(\mathrm{~d}, 3 \mathrm{H}, \mathrm{CH}_{3} \mathrm{CH}, J=6.8 \mathrm{~Hz}\right), 2.04$ $\left(\mathrm{s}, 3 \mathrm{H}, \mathrm{CH}_{3} \mathrm{C}_{\mathrm{Ar}}\right), 2.82-2.92\left(\mathrm{~m}, 1 \mathrm{H}, \mathrm{CH}_{3} \mathrm{CH}\right), 3.09-3.16\left(\mathrm{~m}, 1 \mathrm{H}, \mathrm{CH}_{2} \mathrm{C}_{\mathrm{Ar}}\right), 3.27-3.33(\mathrm{~m}, 1 \mathrm{H}$, $\left.\mathrm{CH}_{2} \mathrm{C}_{\mathrm{Ar}}\right), 3.87-3.96\left(\mathrm{~m}, 1 \mathrm{H}, \mathrm{CH}_{2} \mathrm{Te}\right), 4.04-4.15\left(\mathrm{~m}, 1 \mathrm{H}, \mathrm{CH}_{2} \mathrm{Te}\right), 5.48-5.55(\mathrm{~m}, 1 \mathrm{H}, \mathrm{CHO})$, $6.46\left(\mathrm{~d}, 1 \mathrm{H}, \mathrm{CH}_{\mathrm{Ar}}, J=7.9 \mathrm{~Hz}\right), 6.73\left(\mathrm{~d}, 1 \mathrm{H}, \mathrm{CH}_{\mathrm{Ar}}, J=7.9 \mathrm{~Hz}\right) .{ }^{13} \mathrm{C}-\mathrm{NMR}\left(\mathrm{CDCl}_{3} / \mathrm{DMSO}-d_{6}\right)$ : $18.1\left(\underline{\mathrm{CH}}_{3} \mathrm{C}_{\mathrm{Ar}}\right), 21.9\left(\underline{\mathrm{CH}}_{3} \mathrm{CH}\right), 27.4\left(\mathrm{CH}_{3} \underline{\mathrm{CH}}\right), 34.9\left(\underline{\mathrm{CH}}_{2} \mathrm{C}_{\mathrm{Ar}}\right), 61.5\left(\mathrm{CH}_{2} \mathrm{Te}\right), 79.5(\mathrm{CHO})$, $121.0\left(\mathrm{CH}_{\mathrm{Ar}}\right), 124.2\left(\mathrm{CH}_{2} \underline{\mathrm{C}}_{\mathrm{Ar}}\right), 124.2\left(\mathrm{CH}_{\mathrm{Ar}}\right), 127.1\left(\mathrm{CHC}_{\mathrm{Ar}}\right), 131.5\left(\mathrm{CH}_{3} \underline{\mathrm{C}}_{\mathrm{Ar}}\right), 155.2(\mathrm{C} \mathrm{Ar})$. Anal. calcd for $\mathrm{C}_{13} \mathrm{H}_{17} \mathrm{OBr}_{3} \mathrm{Te}$ (556.58): C 28.05, H 3.08, $\mathrm{Br} 43.07$, Te 22.93\%. Found: C 27.78, H 2.99, Br 43.34, Te 23.17\%.

Tribromo[(4-isopropyl-7-methyl-2,3-dihydro-1-benzofuran-2-yl)methyl]- $\lambda^{4}$-tellane (14) (0.423 g, quantitative yield) was obtained as a light orange powder, mp 59-61 ${ }^{\circ} \mathrm{C}$ (decomp.) from tellurium tetrabromide $(0.27 \mathrm{~g}, 1 \mathrm{mmol})$ and allyl carvacrol $6(0.19 \mathrm{~g}, 1 \mathrm{mmol})$ in acetonitrile under the same conditions as compound 13. ${ }^{1} \mathrm{H}-\mathrm{NMR}\left(\mathrm{CDCl}_{3}\right): 0.95\left(\mathrm{~d}, 3 \mathrm{H}, \mathrm{CH}_{3} \mathrm{CH}\right.$, $J=6.8 \mathrm{~Hz}), 0.96\left(\mathrm{~d}, 3 \mathrm{H}, \mathrm{CH}_{3} \mathrm{CH}, J=6.8 \mathrm{~Hz}\right), 1.89\left(\mathrm{~s}, 3 \mathrm{H}, \underline{\mathrm{CH}}_{3} \mathrm{C}_{\mathrm{Ar}}\right), 2.56-2.67\left(\mathrm{~m}, 1 \mathrm{H}, \mathrm{CH}_{3} \mathrm{C} \underline{\mathrm{H}}\right)$, 3.20-3.26 (m, 2H, CH $\mathrm{CH}_{2} \mathrm{Te}$ 3.81-3.88 (m, 1H, $\left.\mathrm{CH}_{2} \mathrm{C}_{\mathrm{Ar}}\right)$, 3.99-4.03 (m, 1H, $\left.\mathrm{CH}_{2} \mathrm{C}_{\mathrm{Ar}}\right)$, 5.38-5.45 $(\mathrm{m}, 1 \mathrm{H}, \mathrm{CHO}), 6.40\left(\mathrm{~d}, 1 \mathrm{H}, \mathrm{CH}_{\mathrm{Ar}}, J=7.8 \mathrm{~Hz}\right), 6.63\left(\mathrm{~d}, 1 \mathrm{H}, \mathrm{CH}_{\mathrm{Ar}}, J=7.8 \mathrm{~Hz}\right) .{ }^{13} \mathrm{C}-\mathrm{NMR}$ $\left(\mathrm{CDCl}_{3} / \mathrm{DMSO}-d_{6}\right): 14.5\left(\underline{\mathrm{CH}}_{3} \mathrm{C}_{\mathrm{Ar}}\right), 22.2\left(\underline{\mathrm{CH}}_{3} \mathrm{CH}\right), 22.4\left(\mathrm{CH}_{3} \mathrm{CH}\right), 30.6\left(\mathrm{CH}_{3} \underline{\mathrm{CH}}\right), 34.5$ $\left(\underline{\mathrm{CH}}_{2} \mathrm{C}_{\mathrm{Ar}}\right), 61.5\left(\mathrm{CH}_{2} \mathrm{Te}\right), 79.6(\mathrm{CHO}), 116.0\left(\mathrm{CH}_{\mathrm{Ar}}\right), 116.4\left(\mathrm{CH}_{2} \underline{\mathrm{C}}_{\mathrm{Ar}}\right), 122.5\left(\mathrm{CH}_{3} \underline{\mathrm{C}}_{\mathrm{Ar}}\right), 128.7$ $\left(\mathrm{CH}_{\mathrm{Ar}}\right), 142.5\left(\mathrm{CHC} \underline{A r}_{\mathrm{Ar}}\right), 156.2\left(\mathrm{C}_{\mathrm{Ar}} \mathrm{O}\right)$. Anal. calcd for $\mathrm{C}_{13} \mathrm{H}_{17} \mathrm{OBr}_{3} \mathrm{Te}$ (556.58): C 28.05, $\mathrm{H}$ 3.08, Br 43.07, Te 22.93\%. Found: C 28.16, H 3.13, Br 43.29, Te 23.14\%. 
Bis[(7-isopropyl-4-methyl-2,3-dihydro-1-benzofuran-2-yl)methyl] ditelluride (15). A solution of $\mathrm{Na}_{2} \mathrm{~S}_{2} \mathrm{O}_{5}(1.2 \mathrm{~g}, 6.3 \mathrm{mmol})$ in water $(8 \mathrm{~mL})$ was added to a stirred mixture of trichlorotellane $11(0.423 \mathrm{~g}, 1 \mathrm{mmol})$ in methylene chloride $(10 \mathrm{~mL})$. The obtained mixture was stirred at room temperature for $6 \mathrm{~h}$. The organic layer was separated and the aqueous phase was extracted by methylene chloride $(8 \mathrm{~mL})$. The combined organic phase was dried over $\mathrm{CaCl}_{2}$, the solvent was removed by a rotary evaporator and the residue was dried in vacuum. Compound 15 (0.288 g, 91\% yield) was obtained as a dark orange viscous oil. ${ }^{1} \mathrm{H}-\mathrm{NMR}\left(\mathrm{CDCl}_{3}\right): 1.15\left(\mathrm{~d}, 12 \mathrm{H}, \mathrm{CH}_{3} \mathrm{CH}, J=6.9 \mathrm{~Hz}\right), 2.11\left(\mathrm{~s}, 6 \mathrm{H}, \mathrm{CH}_{3} \mathrm{C}_{\mathrm{Ar}}\right), 2.76-2.83(\mathrm{~m}, 2 \mathrm{H}$, $\left.\mathrm{CH}_{2} \mathrm{C}_{\mathrm{Ar}}\right), 2.89-2.98\left(\mathrm{~m}, 2 \mathrm{H}, \mathrm{CH}_{3} \mathrm{CH}\right), 3.18-3.24\left(\mathrm{~m}, 2 \mathrm{H}, \mathrm{CH}_{2} \mathrm{C}_{\mathrm{Ar}}\right), 3.42-3.47$ (m, 2H, $\left.\mathrm{CH}_{2} \mathrm{Te}\right)$, 3.54-3.60 (m, 2H, CH $2 \mathrm{Te}), 4.83-4.90(\mathrm{~m}, 2 \mathrm{H}, \mathrm{CHO}), 6.55\left(\mathrm{~d}, 2 \mathrm{H}, \mathrm{CH}_{\mathrm{Ar}}, J=7.3 \mathrm{~Hz}\right), 6.83(\mathrm{~d}$, $\left.2 \mathrm{H}, \mathrm{CH}_{\mathrm{Ar}}, \mathrm{J}=7.3 \mathrm{~Hz}\right) .{ }^{13} \mathrm{C}-\mathrm{NMR}\left(\mathrm{CDCl}_{3}\right): 11.8\left(\mathrm{CH}_{2} \mathrm{Te}\right), 18.6\left(\mathrm{CH}_{3} \mathrm{C}_{\mathrm{Ar}}\right), 22.3\left(\mathrm{CH}_{3} \mathrm{CH}\right), 22.5$ $\left(\underline{\mathrm{CH}}_{3} \mathrm{CH}\right), 28.2\left(\mathrm{CH}_{3} \underline{\mathrm{CH}}\right), 35.6\left(\underline{\mathrm{CH}}_{2} \mathrm{C}_{\mathrm{Ar}}\right), 83.7(\mathrm{CHO}), 121.5\left(\mathrm{CH}_{\mathrm{Ar}}\right), 124.8\left(\mathrm{CH}_{2} \underline{\mathrm{C}}_{\mathrm{Ar}}\right), 125.0$ $\left(\mathrm{CH}_{\mathrm{Ar}}\right), 127.8\left(\mathrm{CHC}_{\mathrm{Ar}}\right), 131.9\left(\mathrm{CH}_{3} \underline{\mathrm{C}}_{\mathrm{Ar}}\right), 156.2\left(\mathrm{C}_{\mathrm{ArO}} \mathrm{O}\right) .{ }^{125} \mathrm{Te}-\mathrm{NMR}\left(100 \mathrm{MHz}, \mathrm{CDCl}_{3}\right): 77.9$ Anal. calcd for $\mathrm{C}_{26} \mathrm{H}_{34} \mathrm{O}_{2} \mathrm{Te}_{2}$ (633.75): C 49.27, $\mathrm{H}$ 5.41, Te 40.27\%. Found: C 49.49, H 5.46, Te $40.08 \%$.

Bis[(4-isopropyl-7-methyl-2,3-dihydro-1-benzofuran-2-yl)methyl] ditelluride (16) was obtained as a dark orange viscous oil $(0.291 \mathrm{~g}, 92 \%$ yield $)$ from trichlorotellane $12(0.423 \mathrm{~g}$, $1 \mathrm{mmol})$ and $\mathrm{Na}_{2} \mathrm{~S}_{2} \mathrm{O}_{5}(1.2 \mathrm{~g}, 6.3 \mathrm{mmol})$ in the two-phase solvent system of water $(8 \mathrm{~mL})$ and methylene chloride $(10 \mathrm{~mL}) .{ }^{1} \mathrm{H}-\mathrm{NMR}\left(\mathrm{CDCl}_{3}\right): 1.14\left(\mathrm{~d}, 12 \mathrm{H}, \mathrm{CH}_{3} \mathrm{CH}, J=7.0 \mathrm{~Hz}\right), 2.08$ $\left(\mathrm{s}, 6 \mathrm{H}, \mathrm{CH}_{3} \mathrm{C}_{\mathrm{Ar}}\right), 2.71-2.78\left(\mathrm{~m}, 2 \mathrm{H}, \mathrm{CH}_{3} \mathrm{CH}\right), 2.83-2.89\left(\mathrm{~m}, 2 \mathrm{H}, \mathrm{CH}_{2} \mathrm{C}_{\mathrm{Ar}}\right), 3.25-3.32(\mathrm{~m}, 2 \mathrm{H}$, $\left.\mathrm{CH}_{2} \mathrm{C}_{\mathrm{Ar}}\right), 3.40-3.48\left(\mathrm{~m}, 2 \mathrm{H}, \mathrm{CH}_{2} \mathrm{Te}\right), 3.56-3.63\left(\mathrm{~m}, 2 \mathrm{H}, \mathrm{CH}_{2} \mathrm{Te}\right), 4.84-4.91(\mathrm{~m}, 2 \mathrm{H}, \mathrm{CHO}), 6.59$ $\left(\mathrm{d}, 2 \mathrm{H}, \mathrm{CH}_{\mathrm{Ar}}, J=7.7 \mathrm{~Hz}\right), 6.83\left(\mathrm{~d}, 2 \mathrm{H}, \mathrm{CH}_{\mathrm{Ar}}, J=7.7 \mathrm{~Hz}\right) .{ }^{13} \mathrm{C}-\mathrm{NMR}\left(\mathrm{CDCl}_{3}\right): 11.8\left(\mathrm{CH}_{2} \mathrm{Te}\right)$, $15.1\left(\underline{\mathrm{CH}}_{3} \mathrm{C}_{\mathrm{Ar}}\right), 23.0\left(\underline{\mathrm{CH}_{3}} \mathrm{CH}\right), 23.0\left(\underline{\mathrm{CH}_{3}} \mathrm{CH}\right), 31.4\left(\mathrm{CH}_{3} \underline{\mathrm{CH}}\right), 35.7\left(\underline{\mathrm{CH}}_{2} \mathrm{C}_{\mathrm{Ar}}\right), 84.0(\mathrm{CHO})$, $117.1\left(\mathrm{CH}_{2} \underline{\mathrm{C}}_{\mathrm{Ar}}\right), 123.2\left(\mathrm{CH}_{3} \underline{\mathrm{C}}_{\mathrm{Ar}}\right), 128.4\left(\mathrm{CH}_{\mathrm{Ar}}\right), 129.8\left(\underline{\mathrm{CH}}_{\mathrm{Ar}}\right), 143.0\left(\mathrm{CHC}_{\mathrm{Ar}}\right), 157.4\left(\mathrm{C}{ }_{\mathrm{Ar}} \mathrm{O}\right)$. ${ }^{125} \mathrm{Te}-\mathrm{NMR}\left(100 \mathrm{MHz}, \mathrm{CDCl}_{3}\right):$ 84.0. Anal. calcd for $\mathrm{C}_{26} \mathrm{H}_{34} \mathrm{O}_{2} \mathrm{Te}_{2}$ (633.75): C 49.27, $\mathrm{H}$ 5.41, Te 40.27\%. Found: C 49.51, H 5.58, Te 40.53\%.

\section{Conclusions}

There are no works in the literature studying chalcogenocyclofunctionalization reactions of sulfur, selenium and tellurium halides on the same substrates and comparing the reactivity of these electrophilic reagents. The present work described regioselective synthesis of novel functionalized condensed organochalcogen compounds by chalcogenocyclofunctionalization reactions based on chalcogen halides and natural products thymol and carvacrol. The conditions for efficient chalcogenocyclofunctionalization by reactions of selenium dihalides, sulfur dichloride and tellurium tetrahalides with allyl thymol and allyl carvacrol were found. Attempts were made to reveal the distinguishing features of each reaction and to compare the reactivity of sulfur, selenium and tellurium halides in chalcogenocyclofunctionalization.

The reactions of tellurium tetrachloride and tetrabromide with allyl thymol and allyl carvacrol proceeded with high selectivity in a regiospecific manner affording monoadducts in quantitative yield.

The obtained products represent a new family of organochalcogen compounds bearing (7-isopropyl-4-methyl-2,3-dihydro-1-benzofuran-2-yl)methyl and (4-isopropyl-7-methyl2,3-dihydro-1-benzofuran-2-yl)methyl moieties with promising biological activity.

Supplementary Materials: The following are available online: examples of the NMR spectra of the obtained compounds.

Author Contributions: Methodology, M.V.M. (Maxim V. Musalov) and V.A.P.; conceptualization, M.V.M. (Maxim V. Musalov) and V.A.P.; investigation (research experiments on selenocyclofunctionalization reactions), V.A.Y.; investigation (research experiments on the synthesis of organotellurium compounds), M.V.M. (Maria V. Musalova); investigation (research experiments on the synthesis of organosulfur compounds), A.A.M.; investigation (NMR experiments), S.V.Z.; supervision, S.V.A. and V.A.P.; data curation, S.V.A.; writing-original draft preparation, V.A.P. and M.V.M. (Maxim V. Musalov); writing-review and editing, V.A.P. All authors have read and agreed to the published version of the manuscript. 
Funding: The research on selenocyclofunctionalization reactions was funded by Russian Foundation for Basic Research, grant 19-33-90214 Aspiranty.

Institutional Review Board Statement: Not applicable.

Informed Consent Statement: Not applicable.

Data Availability Statement: Data is available in this article and supplementary information.

Acknowledgments: The authors thank the Baikal Analytical Center SB RAS for providing the instrumental equipment for structural investigations.

Conflicts of Interest: The authors declare no conflict of interest.

Sample Availability: Samples of the compounds are not available from the authors.

\section{References}

1. Koehn, F.E.; Carter, G.T. The evolving role of natural products in drug discovery. Nat. Rev. Drug Discov. 2005, 4, 206-220. [CrossRef]

2. Newman, D.J.; Cragg, G.M. Natural products as sources of new drugs over the last 25 years. J. Nat. Prod. 2007, 70, 461-477. [CrossRef]

3. Harvey, A.L. Natural products in drug discovery. Drug Discov. Today 2008, 13, 894-901. [CrossRef] [PubMed]

4. Chhetri, B.K.; Lavoie, S.; Sweeney-Jones, A.M.; Kubanek, J. Recent trends in the structural revision of natural products. Nat. Prod. Rep. 2018, 35, 514-531. [CrossRef]

5. Beghyn, T.; Deprez-Poulain, R.; Willand, N.; Folleas, B.; Deprez, B. Natural compounds: Leads or ideas? Bioinspired molecules for drug discovery. Chem. Biol. Drug Design 2008, 72, 3-15. [CrossRef] [PubMed]

6. D'Andrea, J. Ancient Herbs in the J. Paul Getty Museum Gardens; Getty Publications: Malibu, CA, USA, $1982 ;$ p. 84.

7. Tilford, G.L. Edible and Medicinal Plants of the West; Mountain Press Publishing: Missoula, MT, USA, $1997 ;$ p. 240.

8. Nieto, G. Biological Activities of Three Essential Oils of the Lamiaceae Family. Medicines 2017, 4, 63. [CrossRef] [PubMed]

9. Marchese, A.; Orhan, I.E.; Daglia, M.; Barbieri, R.; Di Lorenzo, A.; Nabavi, S.F.; Gortzi, O.; Izadi, M.; Nabavi, S.M. Antibacterial and Antifungal Activities of Thymol: A Brief Review of the Literature. Food Chem. 2016, 210, 402-414. [CrossRef] [PubMed]

10. Krepker, M.; Shemesh, R.; Danin Poleg, Y.; Kashi, Y.; Vaxman, A.; Segal, E. Active Food Packaging Films with Synergistic Antimicrobial Activity. Food Control 2017, 76, 117-126. [CrossRef]

11. Mezzoug, N.; Elhadri, A.; Dallouh, A.; Amkiss, S.; Skali, N.S.; Abrini, J.; Zhiri, A.; Baudoux, D.; Diallo, B.; El Jaziri, M.; et al. Investigation of the Mutagenic and Antimutagenic Effects of Origanum Compactum Essential Oil and Some of Its Constituents. Mutat. Res. Toxicol. Environ. Mutagen. 2007, 629, 100-110. [CrossRef]

12. Kong, J.; Zhang, Y.; Ju, J.; Xie, Y.; Guo, Y.; Cheng, Y.; Qian, H.; Quek, S.Y.; Yao, W. Antifungal Effects of Thymol and Salicylic Acid on Cell Membrane and Mitochondria of Rhizopus Stolonifer and Their Application in Postharvest Preservation of Tomatoes. Food Chem. 2019, 285, 380-388. [CrossRef] [PubMed]

13. Nagoor Meeran, M.F.; Javed, H.; Al Taee, H.; Azimullah, S.; Ojha, S.K. Pharmacological Properties and Molecular Mechanisms of Thymol: Prospects for Its Therapeutic Potential and Pharmaceutical Development. Front. Pharmacol. 2017, 8, 380. [CrossRef] [PubMed]

14. De Morais, S.M.; Vila-Nova, N.S.; Bevilaqua, C.M.L.; Rondon, F.C.; Lobo, C.H.; Moura, A.D.A.A.N.; Sales, A.D.; Rodrigues, A.P.R.; de Figuereido, J.R.; Campello, C.C.; et al. Thymol and Eugenol Derivatives as Potential Antileishmanial Agents. Bioorg. Med. Chem. 2014, 22, 6250-6255. [CrossRef] [PubMed]

15. Llana-Ruiz-Cabello, M.; Pichardo, S.; Maisanaba, S.; Puerto, M.; Prieto, A.I.; Gutiérrez-Praena, D.; Jos, A.; Cameán, A.M. In Vitro Toxicological Evaluation of Essential Oils and Their Main Compounds Used in Active Food Packaging: A Review. Food Chem. Toxicol. 2015, 81, 9-27. [CrossRef]

16. Llana-Ruiz-Cabello, M.; Gutiérrez-Praena, D.; Pichardo, S.; Moreno, F.J.; Bermúdez, J.M.; Aucejo, S.; Cameán, A.M. Cytotoxicity and Morphological Effects Induced by Carvacrol and Thymol on the Human Cell Line Caco-2. Food Chem. Toxicol. 2014, 64, 281-290. [CrossRef]

17. Llana-Ruiz-Cabello, M.; Maisanaba, S.; Puerto, M.; Prieto, A.I.; Pichardo, S.; Jos, Á.; Cameán, A.M. Evaluation of the Mutagenicity and Genotoxic Potential of Carvacrol and Thymol Using the Ames Salmonella Test and Alkaline, Endo III- and FPG-Modified Comet Assays with the Human Cell Line Caco-2. Food Chem. Toxicol. 2014, 72, 122-128. [CrossRef]

18. Dheer, D.; Singh, D.; Kumar, G.; Karnatak, M.; Chandra, S.; Verma, V.P.; Shankar, R. Thymol Chemistry: A Medicinal Toolbox. Curr. Bioact. Compd. 2019, 15, 454-474. [CrossRef]

19. Rajput, J.D.; Bagul, S.D.; Bendre, R.S. Design, synthesis, biological screenings and docking simulations of novel carvacrol and thymol derivatives containing acetohydrazone linkage. Res. Chem. Intermed. 2017, 43, 4893-4906. [CrossRef]

20. Narkhede, H.P.; More, U.B.; Dalal, D.S.; Mahulikar, P.P. Solid-Supported Synthesis of Bio-active Carvacrol Compounds Using Microwaves. Synth. Commun. 2008, 38, 2413-2418. [CrossRef]

21. Bkhaitan, M.M.; Alarjah, M.; Mirza, A.Z.; Abdalla, A.N.; El-Said, H.M.; Faidah, H.S. Preparation and biological evaluation of metronidazole derivatives with monoterpenes and eugenol. Chem. Biol. Drug Design. 2018, 92, 1954-1962. [CrossRef] 
22. Nostro, A.; Blanco, A.R.; Cannatelli, M.A.; Enea, V.; Flamini, G.; Morelli, I.; Sudano Roccaro, A.; Alonzo, V. Susceptibility of methicillin-resistant staphylococci to oregano essential oil, carvacrol and thymol. FEMS Microbiol. Lett. 2004, 230, $191-195$. [CrossRef]

23. Zhao, J.; Li, Y.; Liu, Q.; Gao, K. Antimicrobial activities of some thymol derivatives from the roots of Inula hupehensis. Food Chem. 2010, 120, 512-516. [CrossRef]

24. El-Miligy, M.M.M.; Hazzaa, A.A.; El-Zemity, S.R.; Al-Kubeisi, A.K. Synthesis of Thymol Derivatives as Potential Non-Irritant Antimicrobial and Insecticidal Agents. Curr. Bioactive Comp. 2019, 15, 125-137. [CrossRef]

25. Mastelic, J.; Jerkovic, I.; Blazevic, I.; Poljak-Blazi, M.; Borovic, S.; Ivancic-Bace, I.; Smrecki, V.; Zarkovic, N.; Brcic-Kostic, K.; Vikic-Topic, D. Comparative Study on the Antioxidant and Biological Activities of Carvacrol, Thymol, and Eugenol Derivatives. J. Agricult. Food Chem. 2008, 56, 3989-3996. [CrossRef]

26. Piombino, C.; Lange, H.; Sabuzi, F.; Galloni, P.; Conte, V.; Crestini, C. Lignosulfonate microcapsules for delivery and controlled release of thymol and derivatives. Molecules 2020, 25, 866. [CrossRef] [PubMed]

27. Bagul, S.D.; Rajput, J.D.; Tadavi, S.K.; Bendre, R.S. Design, synthesis and biological activities of novel 5-isopropyl-2methylphenolhydrazide-based sulfonamide derivatives. Res. Chem. Intermed. 2017, 43, 2241-2252. [CrossRef]

28. Wang, K.; Jiang, S.; Pu, T.; Fan, L.; Su, F.; Ye, M. Antifungal activity of phenolic monoterpenes and structure-related compounds against plant pathogenic fungi. Nat. Prod. Res. 2019, 33, 1423-1430. [CrossRef] [PubMed]

29. Pricopie, A.-I.; Ionu, I.; Marc, G.; Arseniu, A.-M.; Vlase, L.; Grozav, A.; Gaina, L.I.; Vodnar, D.C.; Pirnau, A.; Tiperciuc, B. Design and synthesis of novel 1,3-thiazole and 2-hydrazinyl-1,3-thiazole derivatives as anti-Candida agents: In vitro antifungal screening, molecular docking study and spectroscopic investigation of their binding interaction with bovine serum albumin. Molecules 2019, 24, 3435. [CrossRef] [PubMed]

30. Koshti, S.M.; Patil, P.A.; Patil, C.B.; Patil, A.S. Synthesis and characterization of prodrugs of sulfonamides as an azo derivatives of carvacrol. Pharma. Chem. 2018, 10, 1-15.

31. Sobotta, L.; Lijewski, S.; Dlugaszewska, J.; Nowicka, J.; Mielcarek, J.; Goslinski, T. Photodynamic inactivation of Enterococcus faecalis by conjugates of zinc (II) phthalocyanines with thymol and carvacrol loaded into lipid vesicles. Inorg. Chim. Acta. 2019, 489, 180-190. [CrossRef]

32. Jeankumar, V.U.; Sridevi, J.P.; Matikonda, S.S.; Peddi, S.; Alvala, M.; Yogeeswari, P.; Sriram, D. Identification and StructureActivity Relationship Study of Carvacrol Derivatives as Mycobacterium Tuberculosis Chorismate Mutase Inhibitors. J. Enzyme Inhib. Med. Chem. 2014, 29, 547-554.

33. Talavera-Aleman, A.; Rodriguez-Garcia, G.; Lopez, Y.; Garcia-Gutierrez, H.A.; TorresValencia, J.M.; del Rio, R.E.; Cerda-GarciaRojas, C.M.; Joseph-Nathan, P.; Gomez-Hurtado, M.A. Systematic evaluation of thymol derivatives possessing stereogenic or prostereogenic centers. Phytochem. Rev. 2016, 15, 251-277. [CrossRef]

34. Cherkasov, R.A.; Nizamov, I.S.; Gabdullina, G.T.; Almetkina, L.A.; Shamilov, R.R.; Sofronov, A.V. Dithiophosphoric and Dithiophosphonic Acids and Their Derivatives on the Basis of Thymol: Synthesis and Antimicrobial Activity. Phosphorus Sulfur. Silicon Relat. Elements 2013, 188, 33-35. [CrossRef]

35. Chen, L.C.; Lee, T.H.; Sung, P.J.; Shu, C.W.; Lim, Y.P.; Cheng, M.J.; Kuo, W.L.; Chen, J.J. New thymol derivatives and cytotoxic constituents from the root of Eupatorium cannabinum ssp. asiaticum. Chem. Biodivers. 2014, 11, 1374-1380. [CrossRef]

36. Kulabaş, N.; Tatar, E.; Bingöl Özakpınar, Ö.; Özsavci, D.; Pannecouque, C.; De Clercq, E.; Küçükgüzel, İ. Synthesis and antiproliferative evaluation of novel 2-(4H-1,2,4-triazole-3-ylthio)acetamide derivatives as inducers of apoptosis in cancer cells. Eur. J. Med. Chem. 2016, 121, 58-70. [CrossRef]

37. Rajput, J.D.; Bagul, S.D.; Bendre, R.S. Synthesis, biological activities and molecular docking simulation of hydrazone scaffolds of carvacrol, thymol and eugenol. Res. Chem. Intermed. 2017, 43, 6601-6616. [CrossRef]

38. Tiekink, E.R.T. Therapeutic potential of selenium and tellurium compounds: Opportunities yet unrealized. Dalton Trans. 2012, 41, 6390-6395. [CrossRef] [PubMed]

39. Nogueira, C.W.; Zeni, G.; Rocha, J.B.T. Organoselenium and Organotellurium Compounds: Toxicology and Pharmacology. Chem. Rev. 2004, 104, 6255-6286. [CrossRef] [PubMed]

40. Ruberte, A.C.; Sanmartin, C.; Aydillo, C.; Sharma, A.K.; Plano, D. Development and Therapeutic Potential of Selenazo Compounds. J. Med. Chem. 2020, 63, 1473-1489. [CrossRef] [PubMed]

41. Yu, S.-C.; Kuhn, H.; Daniliuc, C.-G.; Ivanov, I.; Jones, P.G.; du Mont, W.-W. 5-Selenization of salicylic acid derivatives yielded isoform-specific 5-lipoxygenase inhibitors. Org. Biomol. Chem. 2010, 8, 828-834. [CrossRef] [PubMed]

42. Potapov, V.A. Organic diselenides, ditellurides, polyselenides and polytellurides. Synthesis and reactions. In Patai's Chemistry of Functional Groups. Organic Selenium and Tellurium Compounds; Rappoport, Z., Ed.; John Wiley and Sons: Chichester, UK, 2013; Volume 4, pp. 765-843.

43. Braga, A.L.; Rafique, J. Synthesis of biologically relevant small molecules containing selenium. Part B. Anti-infective and anticancer compounds. In Patai's Chemistry of Functional Groups. Organic Selenium and Tellurium Compounds; Rappoport, Z., Ed.; John Wiley and Sons: Chichester, UK, 2013; Volume 4, pp. 1053-1117.

44. Azad, G.K.; Tomar, R.S. Ebselen, a promising antioxidant drug: Mechanisms of action and targets of biological pathways. Mol. Biol. Rep. 2014, 41, 4865-4879. [CrossRef]

45. Woollins, J.D.; Laitinen, R.S. (Eds.) Selenium and Tellurium Chemistry. From Small Molecules to Biomolecules and Materials; Springer: Berlin/Heidelberg, Germany, 2011; p. 334. 
46. Santi, C. (Ed.) Organoselenium Chemistry: Between Synthesis and Biochemistry; Bentham Science Publishers: Sharjah, United Arab Emirates, 2014; p. 563.

47. Mugesh, G.; du Mont, W.W.; Sies, H. Chemistry of Biologically Important Synthetic Organoselenium Compounds. Chem. Rev. 2001, 101, 2125-2180. [CrossRef] [PubMed]

48. Lenardao, E.J.; Santi, C.; Sancineto, L. New Frontiers in Organoselenium Compounds; Springer International Publishing AG: Cham, Switzerland, 2018; 189p.

49. Petragnani, N.; Stefani, H.A.; Valduga, C.J. Recent advances in selenocyclofunctionalization reactions. Tetrahedron 2001, 57, 1411-1448. [CrossRef]

50. Kostić, M.D.; Divac, V.M.; Bugarčić, Z.M. Electrophilic Selenocyclofunctionalization in the Synthesis of Biologically Relevant Molecules. Curr. Org. Chem. 2016, 20, 2606-2619. [CrossRef]

51. Kostić, M.D.; Divac, V.M.; Bugarčić, Z.M. An introduction to the kinetics of the triethylamine-mediated selenocyclofunctionalization of 4-pentenoic acid. J. Mol. Struct. 2019, 1175, 24-27. [CrossRef]

52. Nieto, J.; Andres, C.; Perez-Encabo, A. 7-Endo Selenocyclization reactions on chiral 3-prenyl- and 3-cinnamyl-2-hydroxymethylperhydro1,3-benzoxazine derivatives. A way to enantiopure 1,4-oxazepanes. Org. Biomolec. Chem. 2015, 13, 9118-9126. [CrossRef]

53. Pedrosa, R.; Andres, C.; Mendiguchia, P.; Nieto, J. Diastereoselective Synthesis of Enantiopure Morpholines by Electrophilic Selenium-Induced 6-exo Cyclizations on Chiral 3-Allyl-2-hydroxymethylperhydro-1,3-benzoxazine Derivatives. J. Org. Chem. 2006, 71, 8854-8863. [CrossRef]

54. Stefani, H.A.; Costa, I.M.; Silva, D.D.O.; Menezes, P.H.; Rodrigues, A. Selenocyclofunctionalization of $\beta$-ketoamides: Synthesis of substituted dihydrofurans. Phosphorus Sulfur. Silicon Relat. Elements 2001, 171-172, 395-406. [CrossRef]

55. Saikia, I.; Borah, A.J.; Phukan, P. Use of Bromine and Bromo-Organic Compounds in Organic Synthesis. Chem. Rev. 2016, 116, 6837-7042. [CrossRef]

56. Kloeckner, U.; Finkbeiner, P.; Nachtsheim, B.J. Iodide-Catalyzed Halocyclization/Cycloaddition/Elimination Cascade Reaction. J. Org. Chem. 2013, 78, 2751-2756. [CrossRef]

57. Parker, P.D.; Lemercier, B.C.; Pierce, J.G. Synthesis of Quaternary-Substituted Thiazolines via Halocyclization of S-Allyl Thioimidate Salts. J. Org. Chem. 2018, 83, 12-22. [CrossRef] [PubMed]

58. Accurso, A.A.; Cho, S.-H.; Amin, A.; Potapov, V.A.; Amosova, S.V.; Finn, M.G. Thia-, Aza-, and Selena[3.3.1]bicyclononane Dichlorides: Rates vs Internal Nucleophile in Anchimeric Assistance. J. Org. Chem. 2011, 76, 4392-4395. [CrossRef]

59. Potapov, V.A.; Amosova, S.V.; Belozerova, O.V.; Albanov, A.I.; Yarosh, O.G.; Voronkov, M.G. Synthesis of 3,6-dihalo-4,4-dimethyl1,4-selenasilafulvenes. Chem. Heterocycl. Compd. 2003, 39, 549-550. [CrossRef]

60. Potapov, V.A.; Amosova, S.V. New Methods for Preparation of Organoselenium and Organotellurium Compounds from Elemental Chalcogens. Russ. J. Org. Chem. 2003, 39, 1373-1380. [CrossRef]

61. Abakumov, G.A.; Piskunov, A.V.; Cherkasov, V.K.; Fedushkin, I.L.; Ananikov, V.P.; Eremin, D.B.; Gordeev, E.G.; Beletskaya, I.P.; Averin, A.D.; Bochkarev, M.N.; et al. Organoelement chemistry: Promising growth areas and challenges. Russ. Chem. Rev. 2018, 87, 393-507. [CrossRef]

62. Musalov, M.V.; Potapov, V.A.; Musalova, M.V.; Amosova, S.V. Regioselective synthesis of bis[(2,3-dihydro-1-benzofuran-2yl)methyl]selenide. Russ. J. Org. Chem. 2014, 50, 1702-1703. [CrossRef]

63. Musalov, M.V.; Ishigeev, R.S.; Udalova, S.I.; Musalova, M.V.; Kurkutov, E.O.; Khabibulina, A.G.; Albanov, A.I.; Potapov, V.A.; Amosova, S.V. Synthesis of Fused Compounds on the Basis of Chalcogen Chlorides and 2-Allylphenols. Russ. J. Org. Chem. 2018, 54, 1035-1040. [CrossRef]

64. Kurkutov, E.O.; Potapov, V.A.; Musalov, M.V.; Amosova, S.V. Bis(1,3-dioxolan-2-ylmethyl)selenide. Russ. J. Gen. Chem. 2017, 87, 357-358. [CrossRef]

65. Musalov, M.V.; Potapov, V.A. Selenium dihalides: New possibilities for the synthesis of selenium-containing heterocycles. Chem. Heterocycl. Comp. 2017, 53, 150-152. [CrossRef]

66. Potapov, V.A.; Kurkutov, E.O.; Musalov, M.V.; Amosova, S.V. Effective synthesis of bis(1,4-dioxan-2-ylmethyl)selenide and selenoxide. Russ. J. Org. Chem. 2016, 52, 1715-1716. [CrossRef]

67. Musalov, M.V.; Ishigeev, R.S.; Potapov, V.A.; Amosova, S.V. Reaction of selenium dihalides with 2-(allylsulfanyl)ethanol. Russ. J. Org. Chem. 2016, 52, 1533-1534. [CrossRef]

68. Potapov, V.A.; Kurkutov, E.O.; Musalov, M.V.; Amosova, S.V. Efficient method for the synthesis of bis(tetrahydro-2H-pyran-2ylmethyl) selenide. Russ. Chem. Bull. 2015, 64, 2973-2974. [CrossRef]

69. Potapov, V.A.; Musalov, M.V.; Kurkutov, E.O.; Musalova, M.V.; Albanov, A.I.; Amosova, S.V. Synthesis of new functionalized organoselenium compounds by heterocyclization of selenium dihalides with pent-4-en-1-ol. Russ. J. Org. Chem. 2016, 52, 339-342. [CrossRef]

70. Musalov, M.V.; Yakimov, V.A.; Potapov, V.A.; Amosova, S.V.; Borodina, T.N.; Zinchenko, S.V. A novel methodology for the synthesis of condensed selenium heterocycles based on the annulation and annulation-methoxylation reactions of selenium dihalides. New J. Chem. 2019, 43, 18476-18483. [CrossRef]

71. Musalov, M.V.; Yakimov, V.A.; Potapov, V.A. Synthesis of Functional Dihydro-1,4-benzoxaselenines from Carvacrol Allyl Ether and Selenium Dihalides. Russ. J. Org. Chem. 2020, 56, 2258-2262. [CrossRef]

72. Coleman, M.T.; LeBlanc, G. Use of Diethoxymethane as a Solvent for Phase Transfer-Catalyzed O-Alkylation of Phenols. Org. Proc. Res. Develop. 2010, 14, 732-736. [CrossRef] 
73. Smit, V.A.; Zefirov, N.S.; Bodrikov, I.V.; Krimer, M.Z. Episulfonium ions: Myth and reality. Acc. Chem. Res. 1979, 12, 282-288. [CrossRef]

74. Abu-yousef, I.A.; Harpp, D.N. New Sulfenyl Chloride Chemistry: Synthesis, Reactions and Mechanisms toward Carbon-Carbon Double Bonds. Sulfur. Rep. 2003, 24, 255-282. [CrossRef]

75. Rasteikiene, L.; Greiciute, D.; Lin'kova, M.G.; Knunyants, I.L. The Addition of Sulphenyl Chlorides to Unsaturated Compounds. Russ. Chem. Rev. 1977, 46, 548-564. [CrossRef]

76. Musalov, M.V.; Potapov, V.A.; Kurkutov, E.O.; Musalova, M.V.; Khabibulina, A.G.; Amosova, S.V. Regioselective syntheses of bis(2-haloalkyl) selenides and dihalo[bis(2-haloalkyl)]- $\lambda^{4}$-selanes from selenium dihalides and 1-alkenes, and the methoxyselenenylation reaction. Arkivoc 2017, iii, 365-376. [CrossRef]

77. Potapov, V.A.; Musalov, M.V.; Amosova, S.V. Reactions of selenium dichloride and dibromide with unsaturated ethers. Annulation of 2,3-dihydro-1,4-oxaselenine to the benzene ring. Tetrahedron Lett. 2011, 52, 4606-4610. [CrossRef] 